\title{
Galerkin projected residual method applied to diffusion-reaction problems
}

\author{
Eduardo Gomes Dutra do Carmo ${ }^{\mathrm{a}, *}$, Gustavo Benitez Alvarez ${ }^{\mathrm{b}}$, Fernando Alves Rochinha ${ }^{\mathrm{a}}$, \\ Abimael Fernando Dourado Loula ${ }^{c}$ \\ a COPPE, Universidade Federal do Rio de Janeiro, Ilha do Fundão, P.B. 68509, 21945-970 Rio de Janeiro, RJ, Brazil \\ ${ }^{\mathrm{b}}$ UFF/EEIMVR, Universidade Federal Fluminense, Av. dos Trabalhadores 420, 27225-125 Volta Redonda, RJ, Brazil

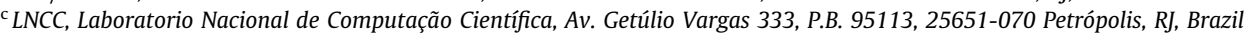

\section{A R T I C L E I N F O}

\section{Article history:}

Received 20 January 2008

Received in revised form 17 May 2008

Accepted 23 May 2008

Available online 10 July 2008

\section{Keywords:}

Stabilization

GLS

GPR

Diffusion-reaction equation

Finite element method

Second-order boundary value problems

\begin{abstract}
A B S T R A C T
A stabilized finite element method is presented for scalar and linear second-order boundary value problems. The method is obtained by adding to the Galerkin formulation multiple projections of the residual of the differential equation at element level. These multiple projections allow the generation of appropriate number of free stabilization parameters in the element matrix depending on the local space of approximation and on the differential operator. The free parameters can be determined imposing some convergence and/or stability criteria or by postulating the element matrix with the desired stability properties. The element matrix of most stabilized methods (such as, GLS and GGLS methods) can be obtained using this new method with appropriate choices of the stabilization parameters. We applied this formulation to diffusion-reaction problems. Optimal rates of convergency are numerically observed for regular solutions.
\end{abstract}

(c) 2008 Elsevier B.V. All rights reserved.

\section{Introduction}

Boundary value problems governed by scalar linear second-order partial differential equations model a great variety of physical phenomena. Usually, the Galerkin finite element method (FEM) is used to solve numerically these boundary value problems. For purely diffusion problems the Galerkin method provides the optimal solution. However, it is well known that most Galerkin finite element methods are unstable and inaccurate for some problems described by scalar and linear second-order partial differential equations, presenting spurious oscillations that do not correspond to the actual solution of the problem.

Advection-diffusion type equation [2-30], diffusion-reaction equation [7,8,31-49] and Helmholtz equation [50-64] are representative examples of the poor stability of the corresponding Galerkin finite element formulations. Stable and accurate numerical solution via finite element method for these problems has been a real challenge, and a great variety of finite element formulations have been developed aiming at stable and accurate approximations. The interested readers should look at [2-64] and references therein. These attempts have used either continuous or discontinuous finite element spaces. The main challenge is to find a consis-

\footnotetext{
* Corresponding author.

E-mail addresses: carmo@con.ufrj.br (E.G. Dutra do Carmo), benitez.gustavo@ gmail.com (G.B. Alvarez), rochinha@adc.coppe.ufrj.br (F.A. Rochinha), aloc@lncc.br (A.F.D. Loula).
}

tent formulation, in continuous or discontinuous finite dimensional spaces, such that its approximate solution is stable and the closest possible to the exact solution in infinite dimensional space ("best approximation"). In that respect, a judicious choice of the stabilization strategy is a decisive issue which has been addressed in most of the above cited papers. In some of them, success has been attained through clever strategies that rely upon automatic choices that explore the local characteristics of the problem. The drawbacks related above motivated us to pursue a new venue for building stable finite element formulations.

Here, we will consider only continuous finite element spaces. Stability and accuracy of finite element methods for these boundary value problems have been consistently improved through the so called stabilized finite element formulations. They are built by adding to the classical Galerkin method different residual terms at the element level which are multiplied by parameters to be tuned to improve stability and accuracy. This is the base of the GLS, GGLS methods [10,31] and other stabilized methods [38] as well. Thus, it seems to be desirable to seek for stabilized methods with a maximum number of free parameters. This method should be built in a such way that, if the criteria to determine the free parameters is the same as adopted, for example, in the GLS, GGLS methods or another linear stabilized method, then the element matrix of these methods can be obtained starting from this new formulation.

In this work, a general formulation is developed for a scalar and linear second-order boundary value problems by adding to the 
Galerkin formulation appropriate multiple projections of residual of the differential equation at element level. These residual projections carry out explicitly the dependence on the differential operator and on the local finite element space. The resulting finite element method can be applied to any space dimension and contains the necessary number of free parameters, determined following some specific criteria to improve the stability and accuracy of the approximate solution. In [64] this method, which we will refer as Galerkin projected residual method (GPR), is applied to the Helmholtz equation with the free parameters of the formulation determined by minimizing the phase error of the approximate solution yielding a $C^{0}$ Lagrangian finite element formulation with minimal pollution.

The paper is organized as follows. The model problem, the associated variational formulation and the corresponding Galerkin finite element approximation are briefly presented in Section 2. In Section 3, we introduce the theoretical base for the GPR method. In section 4 we applied the GPR method to diffusionreaction equation. Some numerical experiments to evaluate the performance of the new formulation are presented in Section 5. Finally, Section 6 contains some conclusions and final remarks.

\section{The model problem}

Let $\Omega \subset \mathbb{R}^{n}(n \geqslant 1)$ be an open bounded domain with a Lipschitz continuous smooth piecewise boundary $\Gamma$. Let $\Gamma_{g}, \Gamma_{q}$ and $\Gamma_{r}$ subsets of $\Gamma$ satisfying meas $\left(\Gamma_{g} \cap \Gamma_{q}\right)=$ meas $\left(\Gamma_{g} \cap \Gamma_{r}\right)=$ meas $\left(\Gamma_{q} \cap \Gamma_{r}\right)=0$ and $\Gamma=\Gamma_{g} \cup \Gamma_{q} \cup \Gamma_{r}$, where meas(.) denotes Lebesgue measure. We denote by $\mathscr{L}(\cdot)$ the scalar and linear second-order differential operator

$\mathscr{L}(\phi)=-\nabla \cdot(D \nabla \phi)+\mathbf{u} \cdot \nabla \phi+\sigma \phi$,

where $\quad D \in H^{1}(\Omega) \cap C^{0}(\Omega \cup \Gamma), \quad \mathbf{u}=\left(u_{1}, \ldots, u_{n}\right) \in\left[H^{1}(\Omega)\right]^{n} \cap$ $\left[C^{0}(\Omega \cup \Gamma)\right]^{n}, \quad \sigma \in C^{0}(\Omega \cup \Gamma), \quad \nabla \phi$ denotes the gradient of $\phi$, $\boldsymbol{\nabla} \cdot(D \boldsymbol{\nabla} \phi)$ denotes the divergent of $D \boldsymbol{\nabla} \phi$ and $\phi \in H^{1, \text { diff }}$ $(D, \Omega)=\left\{\eta \in H^{1}(\Omega) ;(\boldsymbol{\nabla} \cdot(D \nabla \phi)) \in L^{2}(\Omega)\right\} . H^{1}(\Omega)$ and $\left[H^{1}(\Omega)\right]^{n}$ are Sobolev spaces, $C^{0}(\Omega \cup \Gamma)$ is a Banach space, $L^{2}(\Omega)$ is a Hilbert space as defined in Ref. [1]. The dot "." denotes the usual inner product in $\mathbb{R}^{n}$ or in the linear space of matrices, and the derivatives are in the sense of the distributions.

The functions $D$ ("diffusion"), $u$ ("advection") and $\sigma$ ("reaction") are assumed satisfy

$-\frac{\nabla \cdot u}{2} \geqslant v_{o} \geqslant 0$ in $\Omega$ and $0<D_{0} \leqslant D \leqslant \bar{D}$ in $\Omega$,

$\sigma_{0}^{2} \leqslant|\sigma| \leqslant \bar{\sigma}^{2}$ in $\Omega$,

where $D_{0}, \bar{D}, \sigma_{0}, v_{o}$ and $\bar{\sigma}$ are positive real constants.

We consider as our model problem: Find $\phi \in H^{1}(\Omega)$ such that $(\nabla \cdot(D \nabla \phi)) \in L^{2}(\Omega)$ satisfying

$\mathscr{L}(\phi)=f \quad$ in $\Omega$,

$\phi=g$ on $\Gamma_{g}$,

$(D \nabla \phi) \cdot \mathbf{n}=q$ on $\Gamma_{q}$,

$(D \nabla \phi) \cdot \mathbf{n}+\alpha \phi=r \quad$ on $\Gamma_{r}$,

where $f \in L^{2}(\Omega), \quad g \in H^{1 / 2}\left(\Gamma_{g}\right) \cap C^{0}\left(\Gamma_{g}\right), \quad q \in L^{2}\left(\Gamma_{q}\right), \quad r \in L^{2}\left(\Gamma_{r}\right)$, $\alpha \in L^{\infty}\left(\Gamma_{r}\right)$ and the spaces $H^{1 / 2}\left(\Gamma_{g}\right), C^{0}\left(\Gamma_{g}\right), L^{2}\left(\Gamma_{q}\right), L^{2}\left(\Gamma_{r}\right)$ and $L^{\infty}\left(\Gamma_{r}\right)$ are defined as in [1]. The outward normal unit vector defined almost everywhere on $\Gamma$ is denoted by $\mathbf{n}$.

Defining $S=\left\{\eta \in H^{1}(\Omega) ; \eta=g\right.$ on $\left.\Gamma_{g}\right\}$ and $V=\left\{\eta \in H^{1}(\Omega) ; \eta=\right.$ 0 on $\left.\Gamma_{g}\right\}$, the weak form of the boundary value problem defined by (4)-(7) is introduced as: Find $\phi \in S$ satisfying

$A(\phi, \eta)=F(\eta) \quad \forall \eta \in V$ with

$A(\phi, \eta)=\int_{\Omega}[D(\nabla \phi) \cdot(\nabla \eta)+(\mathbf{u} \cdot \nabla \phi) \eta+\sigma \phi \eta] \mathrm{d} \Omega+\int_{\Gamma_{r}} \alpha \phi \eta \mathrm{d} \Gamma$,

$F(\eta)=\int_{\Omega} f \eta \mathrm{d} \Omega+\int_{\Gamma_{q}} q \eta \mathrm{d} \Gamma+\int_{\Gamma_{r}} r \eta \mathrm{d} \Gamma$.

The major challenges in constructing finite element approximations for this model problem is to find a consistent formulation in continuous finite dimensional spaces with an stable approximate solution as close as possible to the correspondent exact solution in infinite dimensional space given by (8)-(10). In the present article we pursue this goal for the reaction-diffusion problem. A similar effort was developed in [64] for the Helmholtz equation.

\section{The GPR finite element framework}

The present section is devoted to establish the theoretical grounds of the proposed methodology along with its characterization as a framework to develop stabilized finite element formulations. A crucial point to be highlighted consists in the way those formulations are designed, combining the choice of a criterium, encoded in a reference matrix, and the tuning of a set of free parameters. This last feature is frequently found in stabilized methods.

\subsection{Theoretical background}

In this section we briefly present the theoretical background for the GPR finite element formulation. For this, we consider $\Omega^{h}=\left\{\Omega_{1}, \ldots, \Omega_{\mathrm{ne}}\right\}$, a partition of $\Omega$ into non degenerated finite elements $\Omega_{e}$, such that $\Omega_{e}$ can be mapped in standard elements by isoparametric mappings and $\Omega_{e} \cap \Omega_{e^{\prime}}=\varnothing$ if $e \neq e^{\prime}$. Moreover, $\Omega \cup \Gamma=\cup_{e=1}^{\text {ne }}\left(\Omega_{e} \cup \Gamma_{e}\right)$, where $\Gamma_{e}$ denotes the boundary of $\Omega_{e}$. We also assume that $D_{e}, \sigma_{e}$ and $f_{e}$, the corresponding restrictions of $D, \sigma$ and $f$ to $\Omega_{e}$, are continuous in $\Omega_{e}$. Let $k \geqslant 1$ be an integer and consider $\mathscr{P}^{k}\left(\Omega_{e}\right)$ the space of polynomials of degree less than or equal to $k$ in the local coordinates.

Let $H^{h, k}=\left\{\eta \in H^{1}(\Omega) ; \eta_{e} \in \mathscr{P}^{k}\left(\Omega_{e}\right)\right\}, \quad S^{h, k}=\left\{\eta \in H^{h, k} ; \eta=g^{h}\right.$ in $\left.\Gamma_{g}\right\}, V^{h, k}=\left\{\eta \in H^{h, k} ; \eta=0\right.$ in $\left.\Gamma_{g}\right\}$ are the finite dimension spaces, and $g^{h}$ the interpolate of $g$.

As previously stated, the GPR formulation is constructed adding to the Galerkin formulation multiple projections of the residual at element level with a free parameter associated with each projection. The number of free parameters depends on the differential operator and the local approximation space. Thus, the maximum number of linearly independent residual projections will depend on properties of operator (such as symmetry, for example) and on the order of polynomial interpolation adopted. These free parameters would then be determined by appropriate criteria for each specific problem, to get more accuracy and stable approximate solutions. To this end, consider for each fixed element $\Omega_{e}$ the space $E_{\mathrm{GPR}}^{0}\left(\Omega_{e}\right)$ defined as

$E_{\mathrm{GPR}}^{0}\left(\Omega_{e}\right)=\left\{\varphi: \Omega_{e} \rightarrow \mathbb{R} ; \varphi=\sum_{i=1}^{\text {npel }} \sum_{j=1}^{\text {npel }} C_{i, j} \widehat{\mathscr{L}}\left(\eta_{i}\right) \mathscr{L}\left(\eta_{j}\right), C_{i, j} \in \mathbb{R}\right\}$,

where npel denotes the number of nodal points of the element $\Omega_{e}$, and $\eta_{i}(i=1, \ldots$, npel $)$ denotes the usual local shape function associated with the nodal point $i$. $\widehat{\mathscr{L}}$ denotes a special operator which is the core of the proposed formulation and do not necessarily have to be linear. The choice of the operator $\widehat{\mathscr{L}}$ will depend on each specific problem. From (11) follows that, $E_{\mathrm{GPR}}^{0}\left(\Omega_{e}\right)$ is the space generated by $\widehat{\mathscr{L}}\left(\eta_{i}\right) \mathscr{L}\left(\eta_{j}\right),(j, i=1, \ldots$, npel $)$, which is supposed to satisfy the following condition. 
Condition 1: The space $E_{\mathrm{GPR}}^{0}\left(\Omega_{e}\right)$ is not coincident to the space $\left\{0_{L^{2}\left(\Omega_{e}\right)}\right\}$, where $0_{L^{2}\left(\Omega_{e}\right)}$ is the null vector of $L^{2}\left(\Omega_{e}\right)$, or equivalently, there must exist $\left(i^{\prime}, j^{\prime}\right) \in\{1, \ldots$, npel $\} \times\{1, \ldots$, npel $\}$ such that $\widehat{\mathscr{L}}\left(\eta_{i^{\prime}}\right) \mathscr{L}\left(\eta_{j^{\prime}}\right) \neq 0$ in $L^{2}\left(\Omega_{e}\right)$.

A base for $E_{\mathrm{GPR}}^{0}\left(\Omega_{e}\right)$ can be obtained using the methodology presented in [64] or Proposition 1 presented in the next subsection. If $\mathscr{N}$ is the dimension of $E_{\mathrm{GPR}}^{0}\left(\Omega_{e}\right)$ then we can identify a basis $\varphi_{1}^{\mathrm{GPR}, e}, \ldots, \varphi_{\mathscr{N}}^{\mathrm{GPR}, e}$ for $E_{\mathrm{GPR}}^{0}\left(\Omega_{e}\right)$, with $\varphi_{l}^{\mathrm{GPR}, e} \in\left\{\widehat{\mathscr{L}}\left(\eta_{i}\right) \mathscr{L}\left(\eta_{j}\right),(i, j) \in\right.$ $\{1, \ldots$, npel $\} \times\{1, \ldots$, npel $\}\},(l=1, \ldots, \mathscr{N})$.

\subsection{An adequate space of matrices for the GPR method}

The basis $\varphi_{1}^{\mathrm{GPR}, e}, \ldots, \varphi_{\mathcal{N}}^{\mathrm{GPR}, e}$ of the $E_{\mathrm{GPR}}^{0}\left(\Omega_{e}\right)$ space will play an important role on the construction of the GPR element matrix. By construction, the GPR method is obtained by adding multiple projections of the residual to the Galerkin formulation at element level. Thus, the element matrix of GPR method is formed by the sum of Galerkin matrix and linear combination of element matrices associated with these multiple residuals. The optimal or nearly optimal GPR matrix is determined by appropriate choice of the scalar parameters of this linear combination, aiming at obtaining more accurate and stable approximate solutions.

Consider $\mathscr{M}^{e}$, the real linear space of all npel $\times$ npel matrices in $\Omega_{e}$ and denote by $A^{\mathrm{GPR}, l, e}(l=1, \ldots, \mathscr{N})$ the set of $\mathscr{N}$ matrices belonging to $\mathscr{M}^{e}$ with $(i, j)$ entry defined as

$$
\begin{aligned}
A_{i j}^{\mathrm{GPR}, l, e} & =\left(\widehat{\mathscr{L}}\left(\eta_{i}\right) \mathscr{L}\left(\eta_{j}\right), \varphi_{l}^{\mathrm{GPR}, e}\right)_{L^{2}\left(\Omega_{e}\right)} \\
(i, j) & \in\{1, \ldots, \text { npel }\} \times\{1, \ldots, \text { npel }\},
\end{aligned}
$$

then the following property of finite dimensional space with inner product follows.

Proposition 1. The functions $\varphi_{1}^{\mathrm{GPR}, e}, \ldots, \varphi_{\mathcal{R}}^{\mathrm{GPR}, e}$ are linearly independent if and only if the matrices $A^{\mathrm{GPR}, l, e}(l=1, \ldots, \mathscr{N})$ are linearly independent

Proof. (a) Assume $\varphi_{1}^{\mathrm{GPR}, e}, \ldots, \varphi_{\mathcal{N}}^{\mathrm{GPR}, e}$ linearly independent.

Consider the linear combination

$\sum_{l=1}^{\mathcal{N}} \lambda_{l} A^{\mathrm{GPR}, l, e}=\mathbf{0}_{\text {matrix }}$

with $\lambda_{l} \in \mathbb{R}(l=1, \ldots, \mathscr{N})$ and $\mathbf{0}_{\text {matrix }}$ denoting the npel $\times$ npel null matrix. From (13) we have

$\sum_{l=1}^{\mathscr{N}} \lambda_{l} A_{i j}^{\mathrm{GPR}, l, e}=0 \quad \forall(i, j) \in\{1, \ldots$, npel $\} \times\{1, \ldots$, npel $\}$

and from (12) and (14) we obtain

$$
\begin{aligned}
& \sum_{l=1}^{\mathscr{N}} \lambda_{l}\left(\widehat{\mathscr{L}}\left(\eta_{i}\right) \mathscr{L}\left(\eta_{j}\right), \varphi_{l}^{\mathrm{GPR}, e}\right)_{L^{2}\left(\Omega_{e}\right)}=0 \\
& \forall(i, j) \in\{1, \ldots, \text { npel }\} \times\{1, \ldots, \text { npel }\}
\end{aligned}
$$

or equivalently

$\left(\widehat{\mathscr{L}}\left(\eta_{i}\right) \mathscr{L}\left(\eta_{j}\right), \sum_{l=1}^{\mathcal{N}} \lambda_{l} \varphi_{l}^{\mathrm{GPR}, e}\right)_{L^{2}\left(\Omega_{e}\right)}=0$

for all $(i, j) \in\{1, \ldots$, npel $\} \times\{1, \ldots$, npel $\}$. From (11) and (16) follows

$\sum_{l=1}^{\mathscr{N}} \lambda_{l} \varphi_{l}^{\mathrm{GPR}, e}=0 \quad$ in $L^{2}\left(\Omega_{e}\right)$.

Since $\varphi_{1}^{\mathrm{GPR}, e}, \ldots, \varphi_{l}^{\mathrm{GPR}, e}$ are linearly independent, from (17) follows

$\lambda_{l}=0 \quad \forall l \in\{1, \ldots, \mathscr{N}\}$,

proving that the matrices $A^{\mathrm{GPR}, l, e}(l=1, \ldots, \mathscr{N})$ are linearly independent. (b) Assume $A^{\mathrm{GPR}, l, e}(l=1, \ldots, \mathscr{N})$ linearly independent.

Consider the linear combination

$\sum_{l=1}^{\mathcal{N}} \lambda_{l} \varphi_{l}^{\mathrm{GPR}, e}=0 \quad$ in $\Omega_{e}$

with $\lambda_{l} \in \mathbb{R}(l=1, \ldots, \mathscr{N})$. Multiplying Eq. (19) by $\widehat{\mathscr{L}}\left(\eta_{i}\right) \mathscr{L}\left(\eta_{j}\right)$, for each pair $((i, j) \in\{1, \ldots$, npel $\} \times\{1, \ldots$, npel $\})$, and integrating in $\Omega_{e}$, from (12) yields

$\sum_{l=1}^{\mathcal{N}} \lambda_{l}\left(\widehat{\mathscr{L}}\left(\eta_{i}\right) \mathscr{L}\left(\eta_{j}\right), \varphi_{l}^{\mathrm{GPR}, e}\right)_{L^{2}\left(\Omega_{e}\right)}=\sum_{l=1}^{\mathcal{N}} \lambda_{l} A_{i j}^{\mathrm{GPR}, l, e}=0$

for all $(i, j) \in\{1, \ldots$, npel $\} \times\{1, \ldots$, npel $\}$, or

$\sum_{l=1}^{\mathscr{N}} \lambda_{l} A^{\mathrm{GPR}, l, e}=\mathbf{0}_{\text {matrix }}$

which imply

$\lambda_{l}=0 \quad \forall l \in\{1, \ldots, \mathscr{N}\}$

since $A^{\mathrm{GPR}, l, e}(l=1, \ldots, \mathscr{N})$ are linearly independent. Consequently, the functions $\varphi_{1}^{\mathrm{GPR}, e}, \ldots, \varphi_{\mathscr{N}}^{\mathrm{GPR}, e}$ are linearly independent.

The previous property allows to choose an appropriate base for the space of matrices $\mathscr{M}^{\mathrm{GPR}, e} \subset \mathscr{M}^{e}$ generated by the GPR method, that is

$\mathscr{M}^{\mathrm{GPR}, e}=\left\{M \in \mathscr{M}^{e} ; M=\sum_{l=1}^{\mathscr{N}} \lambda_{l} A^{\mathrm{GPR}, l, e}, \lambda_{l} \in \mathbb{R}\right\}$.

The above correspondence between the GPR functions and matrices shows to be very convenient from the computational implementation point of view.

\subsection{The Galerkin projected residual method (GPR)}

Consider the real parameters $\tau_{0}^{e}, \tau_{1}^{e}, \ldots, \tau^{e}$, the vector $\tau^{e}=$ $\left(\tau_{1}^{e}, \ldots, \tau_{\mathcal{N}}^{e}\right) \in \mathbb{R}^{\mathcal{N}}$ and the function $W^{e} \in C^{0}\left(\Omega_{e} \cup \Gamma_{e}\right)$ and define the bilinear form

$A^{\mathrm{GPR}, e}\left(\tau^{e}, \phi, \eta\right)=\sum_{l=1}^{\mathcal{N}} \tau_{l}^{e}\left(\mathscr{L}(\phi), \widehat{\mathscr{L}}(\eta) \varphi_{l}^{\mathrm{GPR}, e}\right)_{L^{2}\left(\Omega_{e}\right)}$

on $H^{1, \text { diff }}\left(D_{e}, \Omega_{e}\right) \times H^{1, \text { diff }}\left(D_{e}, \Omega_{e}\right)$ and the linear functional

$F^{\mathrm{GPR}, e}\left(\tau^{e}, \eta\right)=\sum_{l=1}^{\mathcal{N}} \tau_{l}^{e}\left(f, \widehat{\mathscr{L}}(\eta) \varphi_{l}^{\mathrm{GPR}, e}\right)_{L^{2}\left(\Omega_{e}\right)} \quad \forall \eta \in H^{1, \mathrm{diff}}\left(D_{e}, \Omega_{e}\right)$.

The Galerkin projected residual method (GPR) consist of finding $\phi^{h} \in S^{h, k}$ satisfying $\forall \eta^{h} \in V^{h, k}$

$$
\begin{gathered}
A\left(\phi^{h}, \eta^{h}\right)+\sum_{e=1}^{\mathrm{ne}} \tau_{0}^{e}\left(\mathscr{L}\left(\phi_{e}^{h}\right), \mathscr{L}\left(\eta_{e}^{h}\right)\left|W^{e}\right|\right)_{L^{2}\left(\Omega_{e}\right)}+A^{\mathrm{GPR}, e}\left(\tau^{e}, \phi_{e}^{h}, \eta_{e}^{h}\right) \\
=F\left(\eta^{h}\right)+\sum_{e=1}^{\mathrm{ne}} \tau_{0}^{e}\left(f, \mathscr{L}\left(\eta_{e}^{h}\right)\left|W^{e}\right|\right)_{L^{2}\left(\Omega_{e}\right)}+F^{\mathrm{GPR}, e}\left(\tau^{e}, \eta_{e}^{h}\right) .
\end{gathered}
$$

The function $W^{e}$ and the parameter $\tau_{0}^{e}$ will be determined after the parameters $\tau_{1}^{e}, \ldots, \tau_{\mathcal{N}}^{e}$. It is possible to consider the function $W^{e}$ dependent on the parameters $\tau_{1}^{e}, \ldots, \tau^{e}$ and on the base $\varphi_{1}^{\mathrm{GPR}, e}, \ldots, \varphi_{\mathscr{N}}^{\mathrm{GPR}, e}$.

To determine the vector $\tau^{e}=\left(\tau_{1}^{e}, \ldots, \tau_{\mathcal{N}}^{e}\right)$ via an appropriate criterion for each specific problem, we shall determine the element matrix of the GPR method as follows. Considering the restriction of $\phi^{h}$ to $\Omega_{e}$

$\phi_{e}^{h}=\sum_{j=1}^{\text {npel }} \hat{\phi}_{e}^{h}(j) \eta_{j}$ 
where $\hat{\phi}_{e}^{h}(j)$ denotes the value of $\phi_{e}^{h}$ on the local node " $j$ " of $\Omega_{e}$ and $\eta_{j}$ is the standard finite element polynomials restricted to the element $e$ and denoting $A_{i, j}^{\mathrm{G}, e}$ and $A_{i, j}^{\mathrm{GLS}, e}$ as $(i, j)$ - entry of the element matrix for Galerkin and a weighted GLS methods, respectively, we have

$A_{i, j}^{\mathrm{G}, e}=\int_{\Omega_{e}}\left(D \nabla \eta_{j} \cdot \nabla \eta_{i}+\mathbf{u} \cdot \nabla \eta_{j} \eta_{i}+\sigma \eta_{j} \eta_{i}\right) \mathrm{d} \Omega+\int_{\Gamma_{e} \cap \Gamma_{r}} \alpha \eta_{j} \eta_{i} \mathrm{~d} \Gamma$

$A_{i, j}^{\mathrm{GLS}, e}=A_{i, j}^{\mathrm{G}, e}+\tau_{0}^{e}\left(\mathscr{L}\left(\eta_{j}\right), \mathscr{L}\left(\eta_{i}\right)\left|W^{e}\right|\right)_{L^{2}\left(\Omega_{e}\right)}$

and

$\left(\mathscr{L}\left(\phi_{e}^{h}\right), \widehat{\mathscr{L}}\left(\eta_{i}\right) \varphi_{l}^{\mathrm{GPR}, e}\right)_{L^{2}\left(\Omega_{e}\right)}=\sum_{j=1}^{\mathrm{npel}} \hat{\phi}_{e}^{h}(j)\left(\mathscr{L}\left(\eta_{j}\right), \widehat{\mathscr{L}}\left(\eta_{i}\right) \varphi_{l}^{\mathrm{GPR}, e}\right)_{L^{2}\left(\Omega_{e}\right)}$.

From (29) and (12) follows

$\left(\mathscr{L}\left(\phi_{e}^{h}\right), \widehat{\mathscr{L}}\left(\eta_{i}\right) \varphi_{l}^{\mathrm{GPR}, e}\right)_{L^{2}\left(\Omega_{e}\right)}=\sum_{j=1}^{\mathrm{npel}} \hat{\phi}_{e}^{h}(j) A_{i j}^{\mathrm{GPR}, l, e}$.

If $M^{\mathrm{GPR}, e}$ denotes the element matrix of GPR method, then from (12), (24),(26) and (28) follows that its $(i, j)$ - entry denoted by $M_{i, j}^{\mathrm{GPR}, e}$ is given by

$M_{i, j}^{\mathrm{GPR}, e}=A_{i, j}^{\mathrm{GLS}, e}+\sum_{l=1}^{\mathcal{N}} \tau_{l}^{e} A_{i j}^{\mathrm{GPR}, l, e}$.

Note that the GLS method can be considered as a first projection of the residual of the differential equation, while the element matrix of GPR method is formed by the usual part of Galerkin plus $\mathscr{N}+1$ projections of residual of the differential equation at element level including the GLS projection. Moreover, the linear independence of the matrices $A_{i j}^{\mathrm{GPR}, l, e}$ is crucial to add new stabilization terms different of GLS term. The vector $\tau^{e}$ with each component corresponding to a residual projection can be determined through some criterion adopted to improve the accuracy and/or stability of the approximate solution for each specific problem. In general, if we know an appropriate element matrix

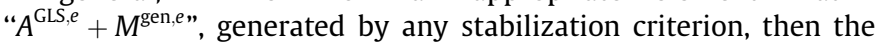
components of the vector $\tau^{e}$ in (31) can be determined, for example, by solving the following minimization problem at element level.

Find $\tau_{1}^{e}, \ldots, \tau_{\mathscr{N}}^{e}$ that minimize the least square functional

$\mathscr{F}\left(M^{\text {gen }, e}\right)=\sum_{i=1}^{\text {npel }} \sum_{j=1}^{\text {npel }}\left[\left(\sum_{l=1}^{\mathcal{N}} \tau_{l}^{e} A_{i j}^{\mathrm{GPR}, l, e}\right)-M_{i, j}^{\mathrm{gen}, e}\right]^{2}$,

or, equivalently

$\frac{\partial \mathscr{F}\left(M^{\text {gen }, e}\right)}{\partial \tau_{k}^{e}}=0 \quad \forall k \in\{1, \ldots, \mathscr{N}\}$

with $M_{i, j}^{\text {gen,e }}$ denoting the $(i, j)$ - entry of $M^{\text {gen }, e}$, which will be named GPR-generating matrix.

Remark 1. A particular GPR method is derived for each specific choice of the set of free parameters $\tau_{l}^{e}$. This set of parameters can be determined, as illustrated above, by knowing or postulating an element matrix with the stability properties coherent with the differential operator. With this strategy in mind, a consistent variational formulation can be derived associated with any postulated element matrix.

Remark 2. The set of parameters can also be determined using information on the solution of the homogeneous or non homogeneous problem like in optimal or nearly optimal Petrov-Galerkin formulations, multiscale or residual free bubble stabilizations.
Remark 3. In [64] a stabilization matrix obtained via standard dispersion analysis for the homogeneous Helmholtz equation is adopted to develop a variationally consistent GPR formulation capable to deal with the non homogeneous equation.

\section{A GPR method for diffusion reaction problems}

From now on, we only consider $\widehat{\mathscr{L}}=\mathscr{L}$ and also restrict our differential model problem to the diffusion-reaction equation, e.g.,

$\mathscr{L}(\phi)=-\nabla \cdot(D \nabla \phi)+\sigma \phi=f$

with the reaction parameter bounded by

$\left(\sigma_{0}\right)^{2} \leqslant \sigma \leqslant(\bar{\sigma})^{2}$ in $\Omega$,

and the boundary conditions given in (5)-(7). As mesh parameter we adopt

$h_{e}=\left(\int_{\Omega_{e}} \mathrm{~d} \Omega\right)^{\frac{1}{n}}$.

Considering that successful stabilized finite element methods have been already applied to reaction diffusion problems, such as the gradient Galerkin least squares (GGLS) and the unusual stabilization (USFEM), we design our method departing from a nontrivial combination of both. Indeed, the possibility of directly combining two stabilizing formulations was explored in [64-66] aiming at obtaining their best features. The possibility of combining stabilization terms is also explored with success in the realm of fluid mechanics in [29]. Here we built the GPR matrix through the weighted stiffness matrix inspired on the GGLS method

$K_{i j}^{e}=\int_{\Omega_{e}} \chi^{e, 2} \sigma\left(J\left(\nabla \eta_{j}\right)\right) \cdot\left(J\left(\nabla \eta_{i}\right)\right) \mathrm{d} \Omega$

and also using the stabilization matrix of the USFEM method to introduce the weighted mass matrix

$M_{i j}^{e}=-\int_{\Omega_{e}} \chi^{e, 1} \sigma \eta_{j} \eta_{i} \mathrm{~d} \Omega$,

where $\chi^{e, 1}$ and $\chi^{e, 2}$ are dimensionless functions, understood as the weights of the nontrivial combination mentioned above, and $J$ is the Jacobian matrix corresponding to the mapping between reference and actual elements. The resulting GPR-generating matrix was adopted to reproduce the ability of the GGLS method in capturing thin sharp layers along with the stable behavior obtained by the USFEM when applied to problems where those layers are no longer confined to thin regions. Indeed, as will be confirmed by the numerical experiments reported in the next section, by exploring this combination the GPR method developed here achieves optimal convergence even in the presence of sharp gradients. Fig. 1 presents numerical results illustrating typical instability of Galerkin approximations for a predominantly reactive diffusion-reaction problem. We clearly observe the spurious oscillations close to the boundary layers compared to the nodally exact solution presented in Fig. 2. The well known GGLS stabilization is capable of reducing these oscillations as shown in Figs. 3 and 4. We also observe the improved performance of GGLS method with bilinear elements (Fig. 3) compared to linear elements (Fig. 4).

It is also worth mentioning that the expression inspired on the GGLS method was used above as, indeed, (37) differs fundamentally from the original form of the stabilizing GGLS term due to the presence of the Jacobian $J$ replacing the $h^{2}$ in order to handle distorted elements. This is also confirmed by the numerical tests. Considering the definition of the Jacobian matrix $J$, we observe that $K_{i j}^{e}$ can be equivalently given by 


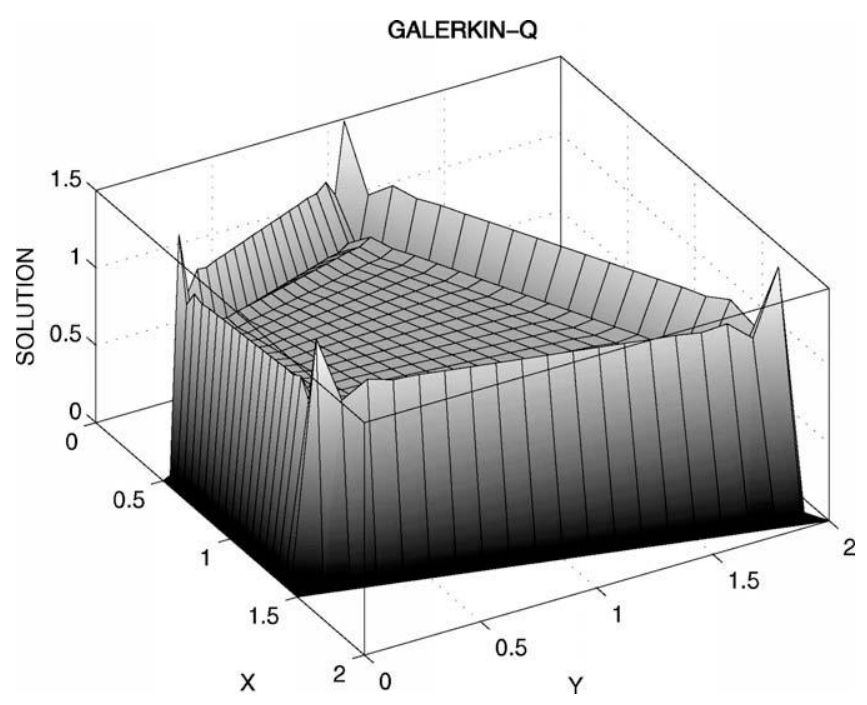

Fig. 1. Galerkin - bilinear elements.

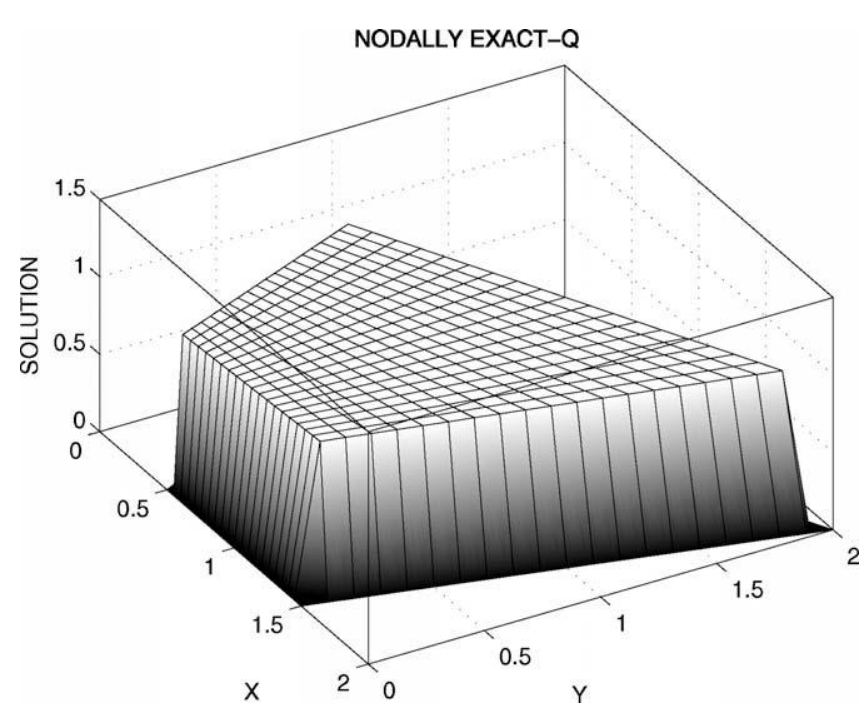

Fig. 2. Nodally exact.

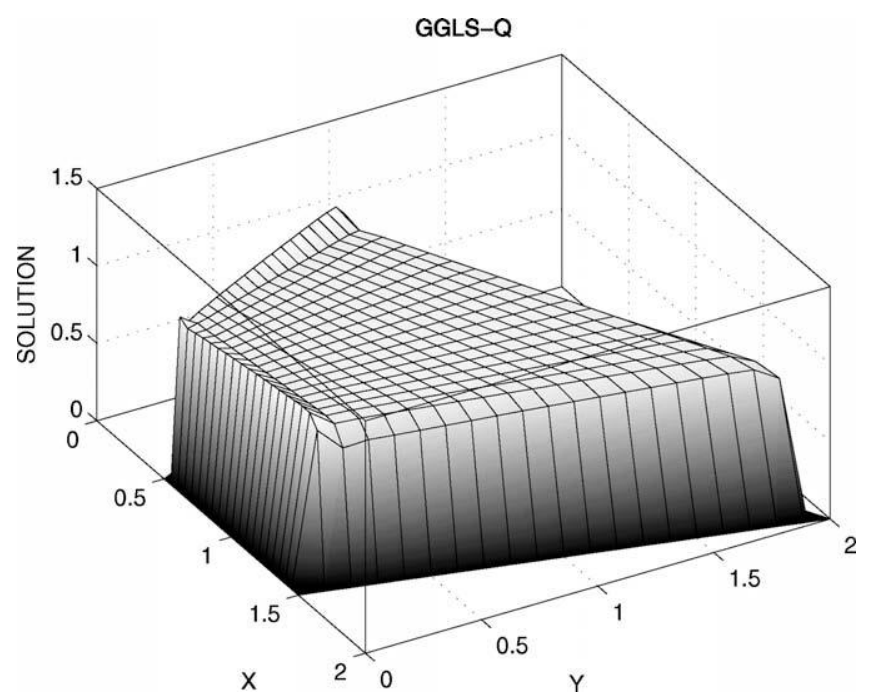

Fig. 3. GGLS - bilinear elements.

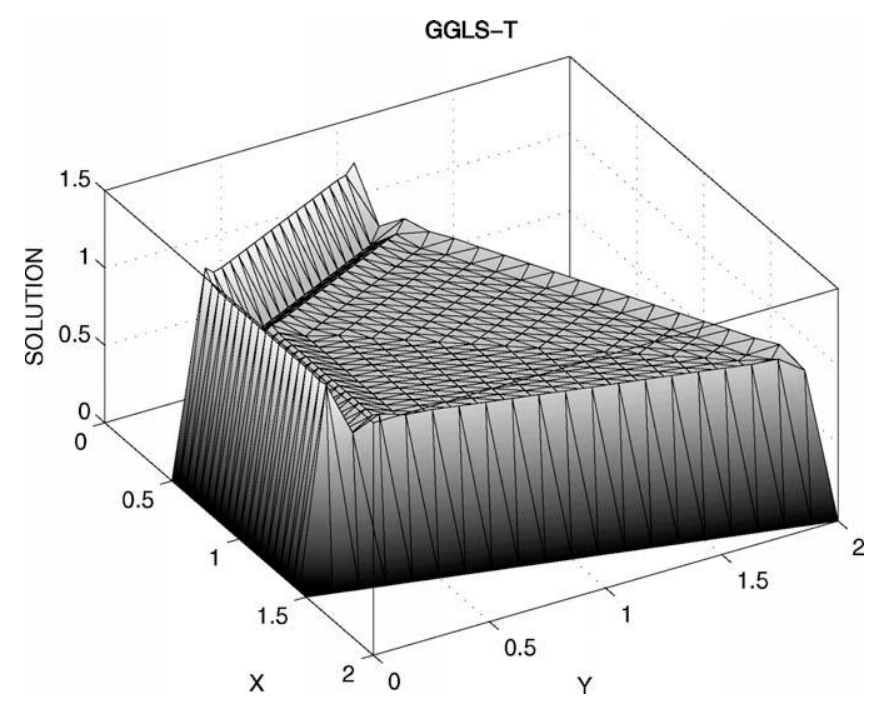

Fig. 4. GGLS - linear elements.

$K_{i j}^{e}=\int_{\Omega_{e}} \chi^{e, 2} \sigma\left(J J^{-1} \nabla_{\mathrm{loc}} \eta_{j}\right) \cdot\left(J J^{-1} \nabla_{\mathrm{loc}} \eta_{i}\right) \mathrm{d} \Omega$,

$K_{i j}^{e}=\int_{\Omega_{e}} \chi^{e, 2} \sigma \nabla_{\mathrm{loc}} \eta_{j} \cdot \nabla_{\mathrm{loc}} \eta_{i} \mathrm{~d} \Omega$

with

$\nabla_{\text {loc }} \eta=\left(\frac{\partial \eta}{\partial \xi_{1}}, \ldots, \frac{\partial \eta}{\partial \xi_{n}}\right)$

for quadrilateral elements and hexahedron elements, and

$\nabla_{\text {loc }} \eta=\left(\frac{\partial \eta}{\partial L_{1}}-\frac{\partial \eta}{\partial L_{n_{t}}}, \ldots, \frac{\partial \eta}{\partial L_{n}}-\frac{\partial \eta}{\partial L_{n_{t}}}\right)\left(n_{t}=n+1\right)$

for triangular elements and tetrahedral elements.

We will consider the simple version (without the term GLS and with $\widehat{\mathscr{L}}=\mathscr{L}$ ) of the GPR method: Find $\phi^{h} \in S^{h, k}$ satisfying $\forall \eta^{h} \in V^{h, k}$

$A\left(\phi^{h}, \eta^{h}\right)+\sum_{e=1}^{\mathrm{ne}} A^{\mathrm{GPR}, e}\left(\tau^{e}, \phi_{e}^{h}, \eta_{e}^{h}\right)=F\left(\eta^{h}\right)+\sum_{e=1}^{\mathrm{ne}} F^{\mathrm{GPR}, e}\left(\tau^{e}, \eta_{e}^{h}\right)$

with the bilinear form $A(\cdot, \cdot)$ and linear functional $F(\cdot)$, corresponding to the Galerkin projection, as defined before and

$A^{\mathrm{GPR}, e}\left(\tau^{e}, \phi, \eta\right)=\sum_{l=1}^{\mathcal{N}} \tau_{l}^{e}\left(\mathscr{L}(\phi), \mathscr{L}(\eta) \varphi_{l}^{\mathrm{GPR}, e}\right)_{L^{2}\left(\Omega_{e}\right)}$,
$F^{\mathrm{GPR}, e}\left(\tau^{e}, \eta\right)=\sum_{l=1}^{\mathcal{N}} \tau_{l}^{e}\left(f, \mathscr{L}(\eta) \varphi_{l}^{\mathrm{GPR}, e}\right)_{L^{2}\left(\Omega_{e}\right)}$

For the elements $\Omega_{e}$ such that $M^{\text {gen,e }} \neq \mathbf{0}_{\text {matrix }}$ the components of the vector $\tau^{e}=\left(\tau_{1}^{e}, \ldots, \tau_{\mathcal{N}}^{e}\right)$ are determined as being the solution of the minimization problem given by (32) and (33) considering the matrix $M^{\text {gen, } e}$ given by

$M^{\text {gen,e }}=K^{e}+M^{e}$

with

$\chi^{e, 1}=\varsigma^{e, 0} \chi^{e}\left(\left|1-\chi^{e}\right|\right)^{\left(\frac{1}{1-\chi^{e}}\right)}$,
$\chi^{e, 2}=\left(\chi^{e}\right)^{\left(\frac{1}{\chi^{e}}\right)} \varsigma^{e, 2} \varsigma^{e, 0}$,

for

$\chi^{e}=\frac{1}{\chi^{e, 0}\left(P_{\text {reat }}^{e}\right)+P_{\text {reat }}^{e}}$, 
where

$P_{\text {reat }}^{e}=\frac{6 D}{\sigma\left(h_{e}\right)^{2}}$

and

$\chi^{e, 0}\left(P_{\text {reat }}^{e}\right)=\left\{\begin{array}{l}1 \text { if } P_{\text {reat }}^{e} \leqslant 1, \\ P_{\text {reat }}^{e} \text { if } P_{\text {reat }}^{e}>1 .\end{array}\right.$

We still need to determine the real constant $\varsigma^{e, 0}$ and the dimensionless function $\varsigma^{e, 2}$. To this end, for each $\Omega_{e}$ and for each $\Omega_{e^{\prime}}$ we consider $[\Psi]_{e e^{\prime}}\left(\Psi \in L^{2}(\Omega)\right.$ and $\left.\Psi_{e} \in H^{1}\left(\Omega_{e}\right) \forall \Omega_{e}\right)$ being defined as follows:

$[\varphi]_{e e^{\prime}}=\int_{\Gamma_{e} \cap \Gamma_{e^{\prime}}}\left|\varphi_{e}-\varphi_{e^{\prime}}\right| \mathrm{d} \Gamma$,

which keeps track of possible discontinuities across element edges. We also introduce $\Gamma_{\text {int }}$ defined as

$\Gamma_{\text {int }}=\bigcup_{e=1}^{\text {ne }}\left(\bigcup_{e^{\prime}=1}^{\mathrm{ne}} \Gamma_{e e^{\prime}}^{*}\right)$,

where

$\Gamma_{e e^{\prime}}^{*}=\Gamma_{e^{\prime} e}^{*}=\left\{\begin{array}{l}\left(\Gamma_{e} \cap \Gamma_{e^{\prime}}\right) \text { if }\left([f]_{e e^{e^{\prime}}} \neq 0 \text { or }[\sigma]_{e e^{\prime}} \neq 0 \text { or }[D]_{e e^{\prime}} \neq 0\right), \\ \varnothing \quad \text { if }\left([f]_{e e^{\prime}}=0 \text { and }[\sigma]_{e e^{\prime}}=0 \text { and }[D]_{e e^{\prime}}=0\right),\end{array}\right.$

which is the union of the external boundary with the internal edges between two elements presenting discontinuous properties or sources. It should be observed that for diffusion-reaction problems, sharp layers will only occur inside an element $\Omega_{e}$ if $\Gamma_{e} \cap\left(\Gamma \cup \Gamma_{\text {int }}\right) \neq$ $\varnothing$.

Based on this observation and inspired on Refs. [31,38], we accomplished a large number of computational experiments with bilinear rectangular elements and linear triangular elements and conclude that the following expressions for the real constant $\varsigma^{e, 0}$ and the dimensionless function $\varsigma^{e, 2}$ present very good stability and accuracy properties

$\varsigma^{e, 0}= \begin{cases}0 & \text { if }\left(\left(\Gamma \cup \Gamma_{\text {int }}\right) \cap \Gamma_{e}\right)=\varnothing, \\ 1 & \text { if }\left(\left(\Gamma \cup \Gamma_{\text {int }}\right) \cap \Gamma_{e}\right) \neq \varnothing,\end{cases}$

$\varsigma^{e, 2}=\left\{\begin{array}{c}\varsigma^{e, q} \text { (if } \Omega_{e} \text { is a quadrilateral element } \\ \text { or is a hexahedron element), } \\ \varsigma^{e, t} \text { (if } \Omega_{e} \text { is a triangular element } \\ \text { or is a tetrahedral element), }\end{array}\right.$

where $\varsigma^{e, q}$ for bilinear quadrilateral element and trilinear hexahedron element and $\varsigma^{e, t}$ for linear triangular element and linear tetrahedral element are, respectively, data as follows:

$\varsigma^{e, q}=\left\{\begin{array}{l}2 / 3+\left(N_{\text {face }}^{e}-1\right) / 6 \text { if meas } \Gamma \cap \Gamma_{e}>0, \\ 2 / 3 \text { if meas }\left(\Gamma \cap \Gamma_{e}\right)=0,\end{array}\right.$

$\varsigma^{e, t}=\left\{\begin{array}{l}\varsigma^{e, q} \quad \text { if }\left(\Gamma \cup \Gamma_{\text {int }}\right) \cap \Gamma_{e}=\varnothing, \\ \left(P_{\text {reat }}^{e}\right)^{-\left(\chi^{e}+1\right)} \text { if }\left(\Gamma \cup \Gamma_{\text {int }}\right) \cap \Gamma_{e} \neq \varnothing\end{array}\right.$

with $N_{\text {face }}^{e}$ being the number of faces of $\Omega_{e}$ contained in $\Gamma$.

Remark 4. From (55) we observe that the extra computational effort demanded by the proposed GPR formulation compared to the Galerkin method is not significant as it corresponds basically to the calculations of the stabilization matrices and forcing vectors of the elements $\Omega_{e}$ such that $\Gamma_{e} \cap\left(\Gamma \cup \Gamma_{\text {int }}\right) \neq \varnothing$. It must be emphasized that those elements are mapped beforehand, which significatively reduces the computational burden.

Remark 5. For GPR finite element approximations with polynomials of degree bigger than 1, additional numeric experiments need to be accomplished to validate the proposed expressions for $\varsigma^{e, q}$ and $\varsigma^{e, t}$.

\section{Numerical results}

To assess the overall performance of the proposed GPR method, a comprehensive number of numerical tests were carried out. Special emphasis was placed in sharp layers and distorted meshes, which often are present on real applications. We will only describe, along their corresponding results, the most significant numeric experiments.

The assessment of our method was accomplished through the use of examples with exact solutions, and comparisons with well

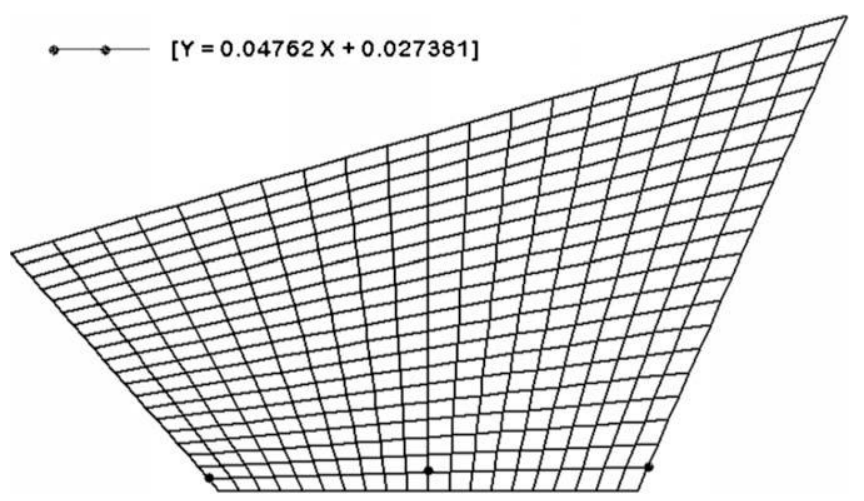

Fig. 5. Nonuniform mesh - quadrilaterals.

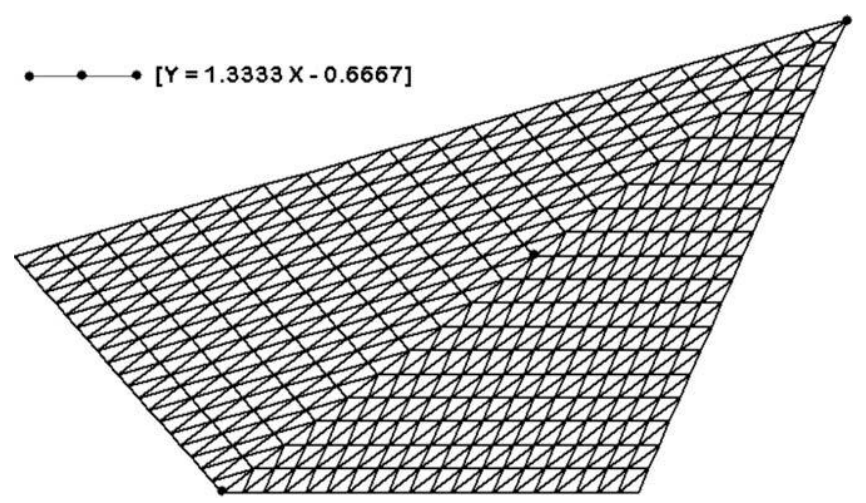

Fig. 6. Nonuniform mesh - triangles.

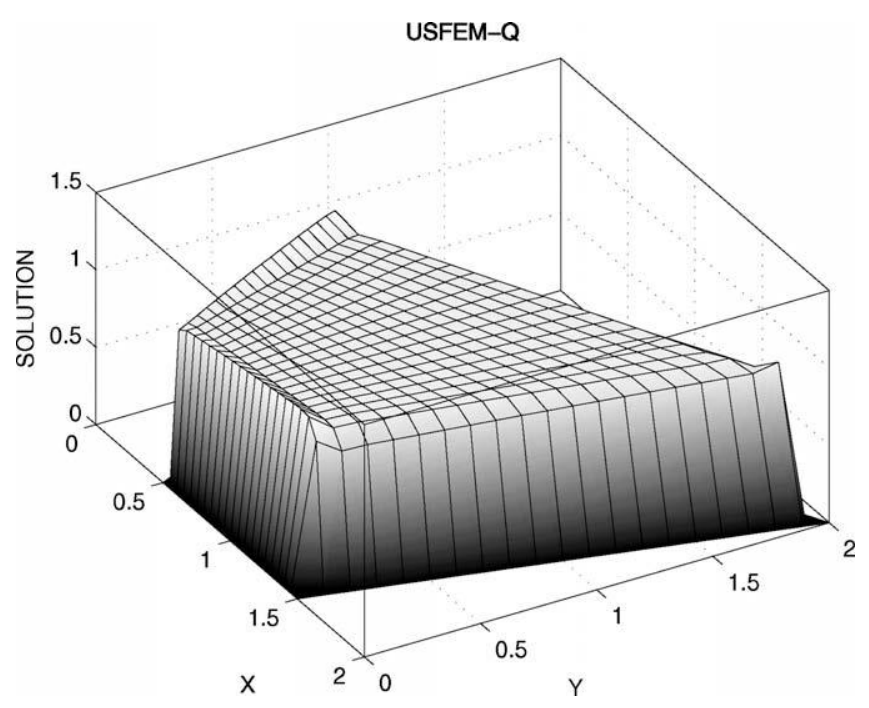

Fig. 7. USFEM - bilinear elements. 


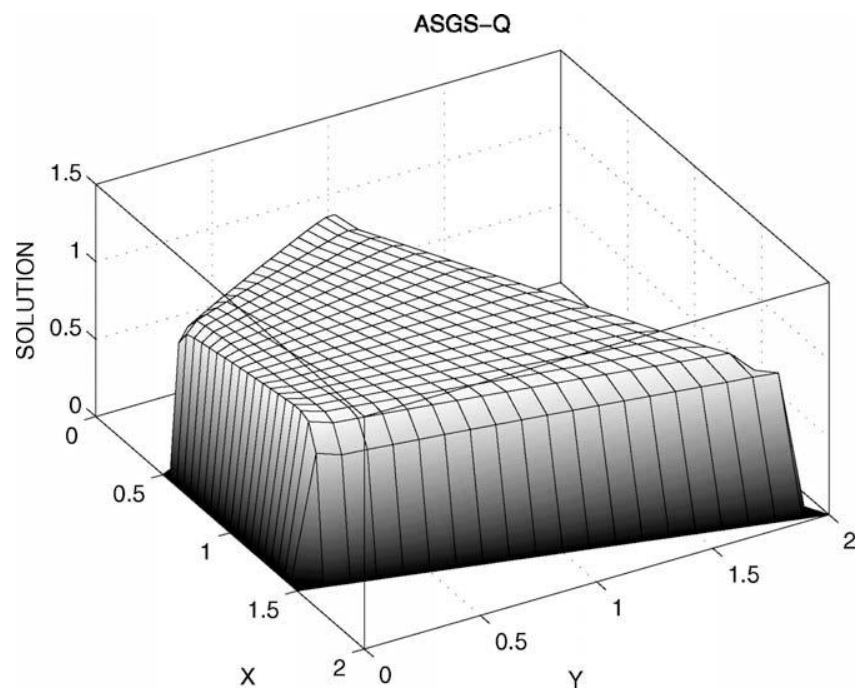

Fig. 8. ASGS - bilinear elements.

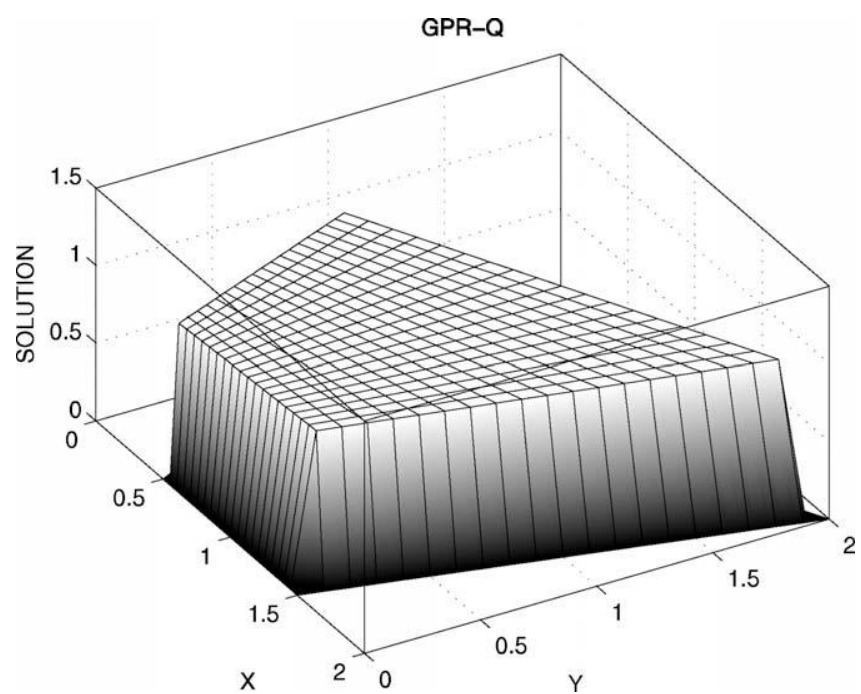

Fig. 9. GPR - bilinear elements.

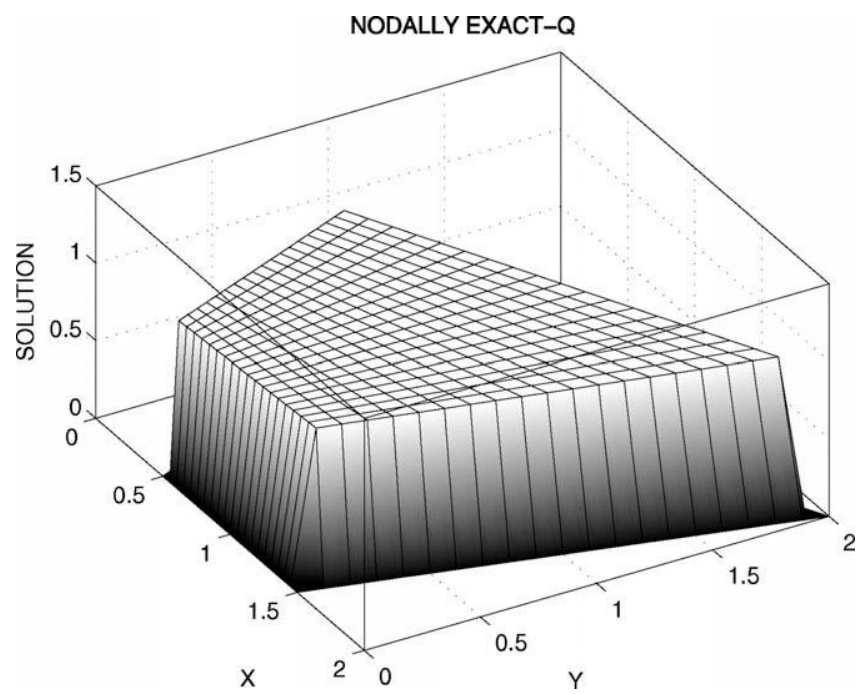

Fig. 10. Nodally exact.

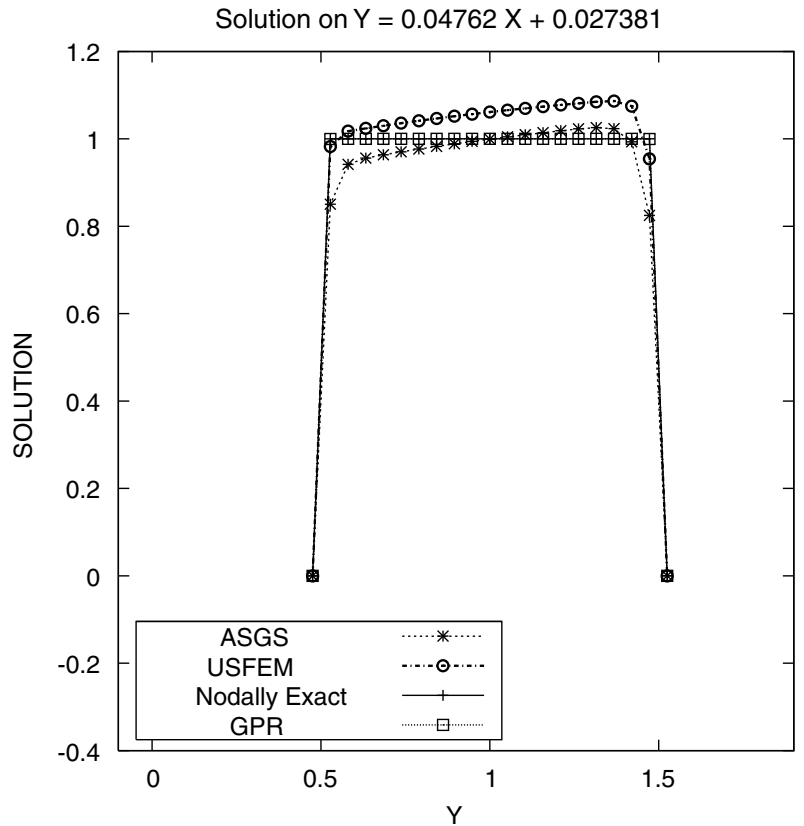

Fig. 11. Bilinear - inclined section.

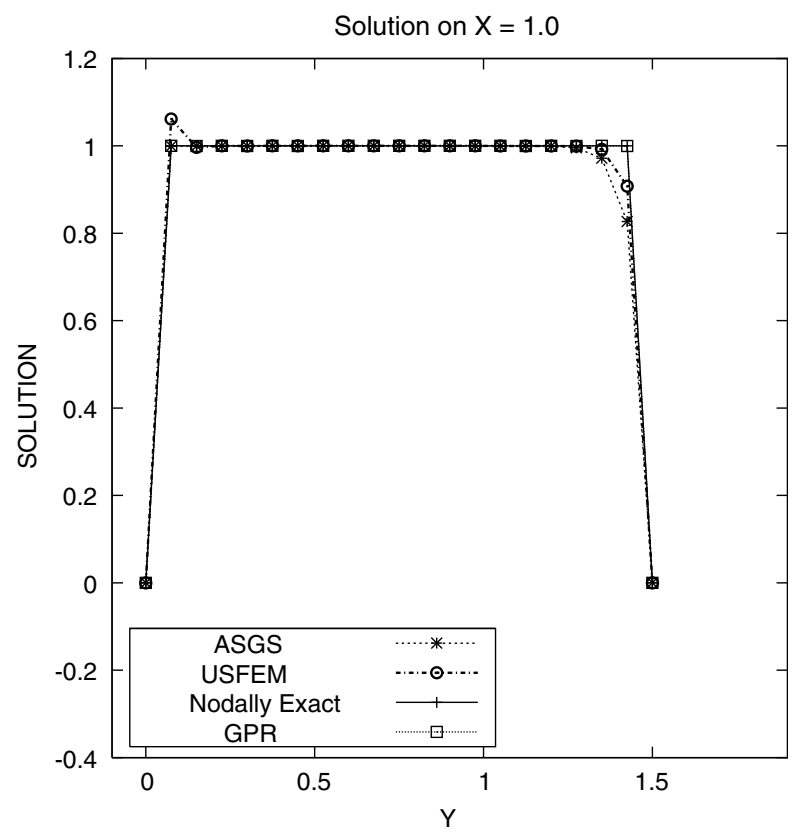

Fig. 12. Bilinear - vertical section.

known stabilized formulations were also considered here. Those comparisons are not intended to be exhaustive through encompassing all methods dealing with stabilized formulations. Only some amid those focused on the diffusion-reaction problem, which in their turn have been already compared to many others, were used to support the conclusions concerning the good performance of the method introduced here. We will denote by "EMM" (enriched multiscale method) the method presented in reference [46], "USFEM" (unusual stabilization) the method presented in [38] and "ASGS" (algebraic subgrid scales) the method introduced in $[37,43]$. 


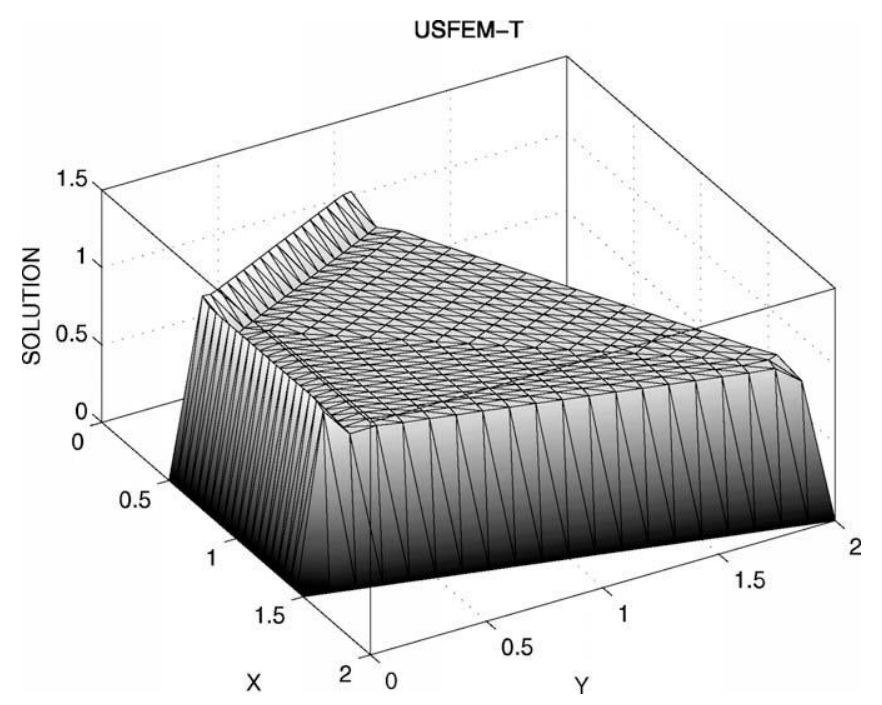

Fig. 13. USFEM - linear elements.

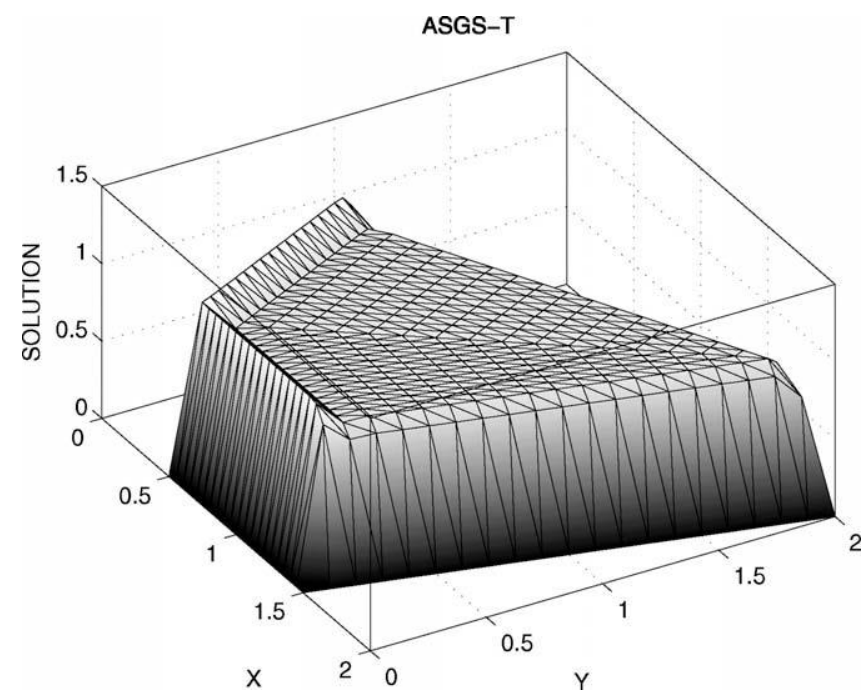

Fig. 14. ASGS - linear elements.

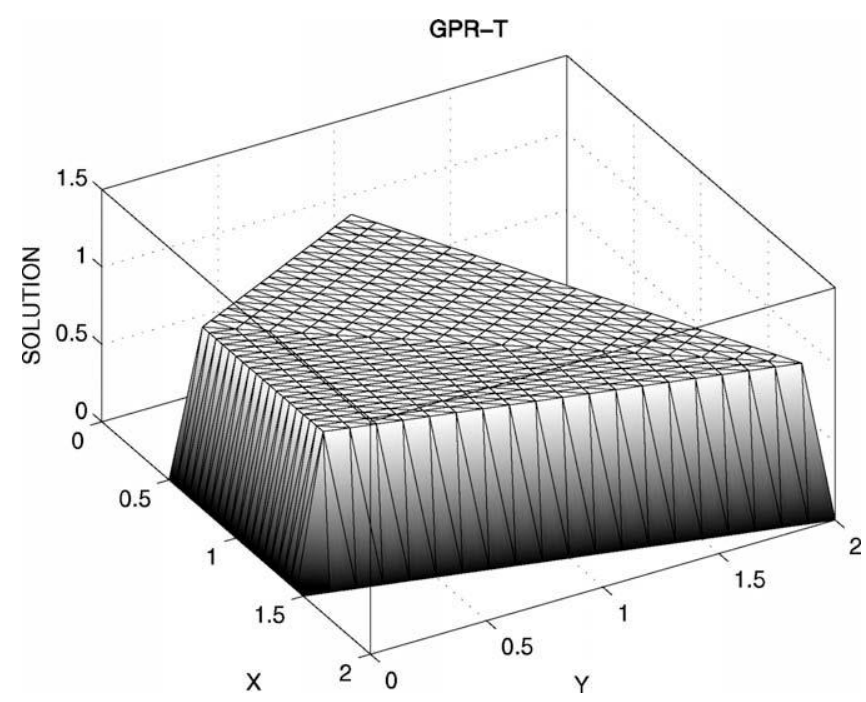

Fig. 15. GPR - linear elements.

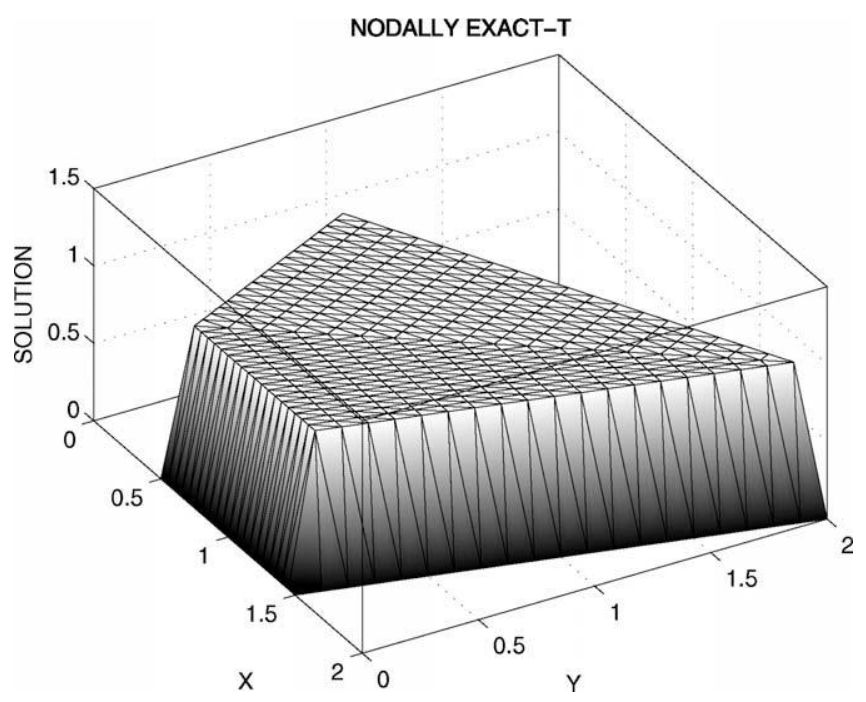

Fig. 16. Nodally exact.

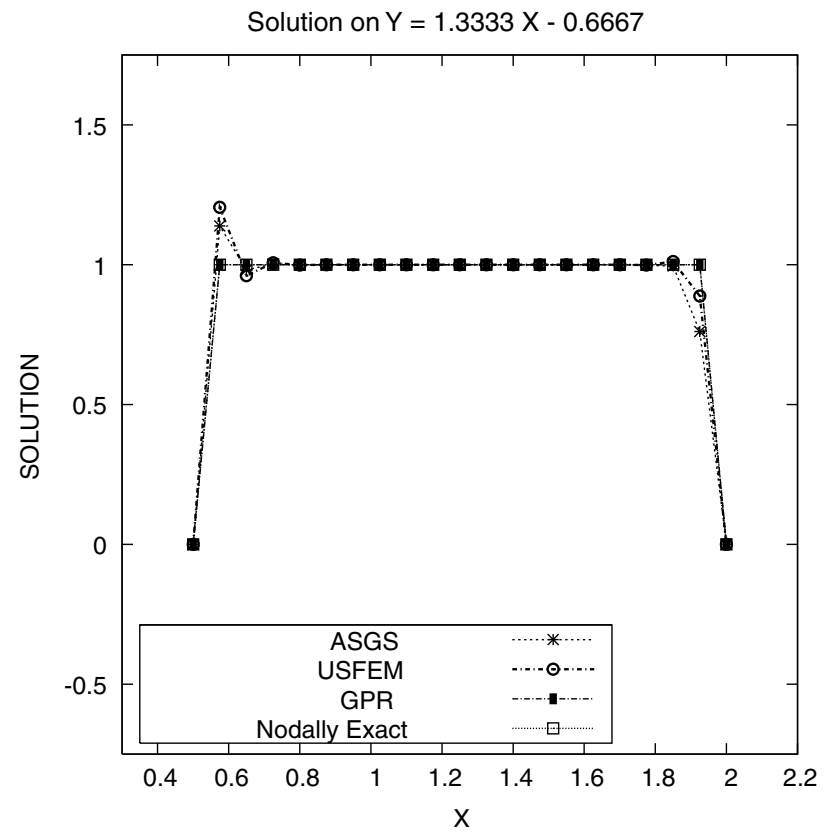

Fig. 17. Linear - inclined section.

\subsection{Quadrilateral domain using non uniform meshes}

This experiment demonstrates the performance of GPR method when applied to a reaction dominated problem defined over a quadrilateral domain of vertices $(0.5,0.0),(1.5,0.0),(2.0,2.0)$ and $(0.0,1.0)$ with $D=10^{-6}, \sigma=1, f=1$ and homogeneous Dirichlet boundary conditions.

Special emphasis is placed on the use of non uniform meshes. Results were obtained for the mesh of quadrilateral elements shown in the Fig. 5 and for the mesh of triangular elements shown in the Fig. 6. Results in 3D plots for "USFEM", "ASGS", "GPR" and nodally exact solutions are presented in Figs. 7-10, respectively, for the mesh of $20 \times 20$ quadrilateral elements. As Ref. [46] does not present the corresponding formulation for distorted quadrilateral elements, we did not present results for the method denominated "EMM". Clearly, we can observe the great performance of the "GPR" method, showing that the effects of the distortion on 
the elements do not cause loss of accuracy and stability, maintaining the accuracy and the stability observed with uniform meshes. However, the effect of the distortion on the elements is clearly observed for the methods "USFEM" and "ASGS", with evident losses of accuracy and stability. Figs. 11 and 12 present 2D plots comparing these solutions in two sections.

Similar results in 3D plots are presented in Figs. 13-16 for the mesh of 800 triangular elements. Once more, we can observe the great accuracy and stability of the methods "GPR" and presenting equivalent performance, and indicating again that the effects of the distortion on the elements do not cause loss of accuracy and stability. Again, it is clear the effect of the distortion on the

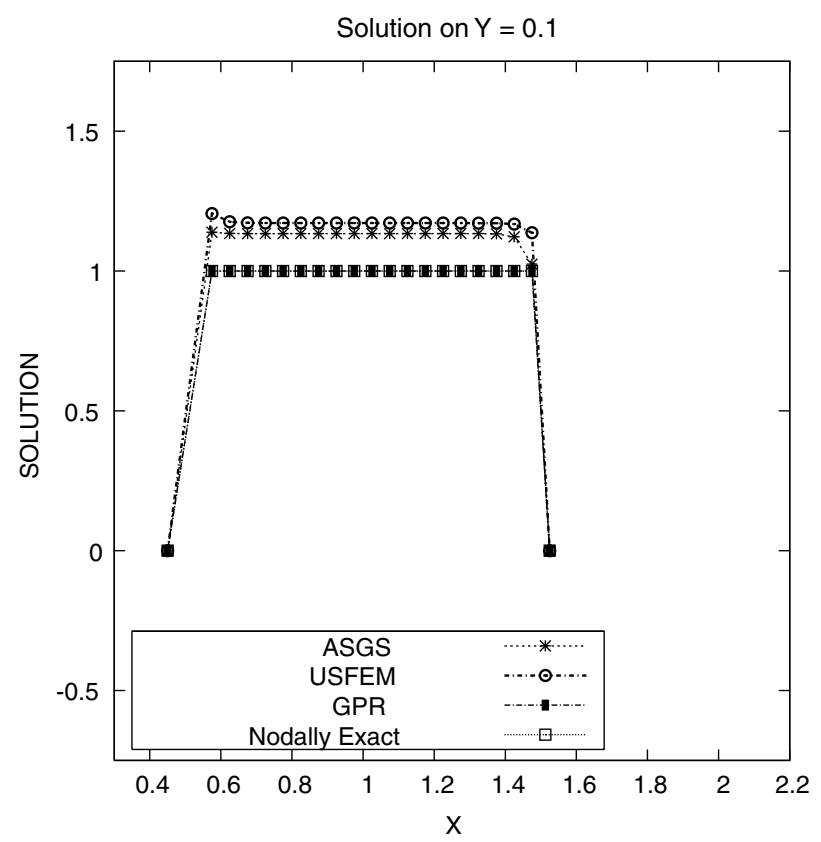

Fig. 18. Linear - horizontal section.

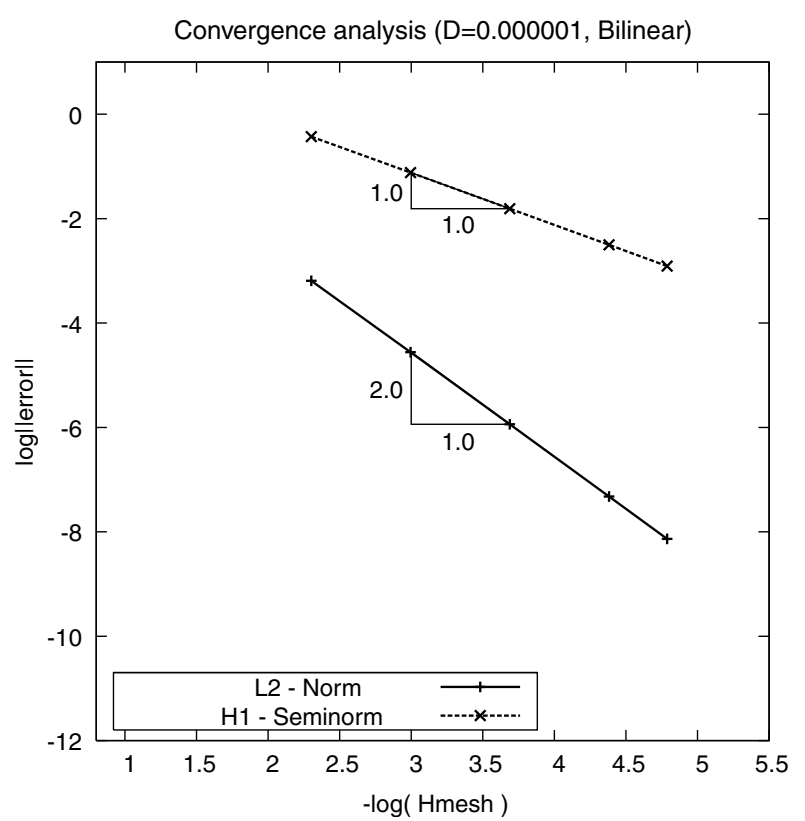

Fig. 19. Bilinear elements. elements for the methods "USFEM" and "ASGS", with remarkable losses of accuracy and stability. In this case the "EMM" method, not shown here, presents performance to the "GPR" method. Figs. 17 and 18 present 2D plots comparing these solutions in two sections.

\subsection{Convergence study}

The second numerical experiment consists of obtaining the convergence rates expressed in terms of $L^{2}(\Omega)$ and $H^{1}(\Omega)$ norms for the problem defined over the quadrilateral domain given in Section 5.1 above, with $\sigma=1, f=\left(2 \pi^{2} D+1\right) \sin (\pi x) \sin (\pi y)$ and boundary conditions $\phi=\sin (\pi x) \sin (\pi y)$ on $\Gamma$. The results were obtained for quadrilateral meshes with partitions $10 \times 10,20 \times 20,40 \times$

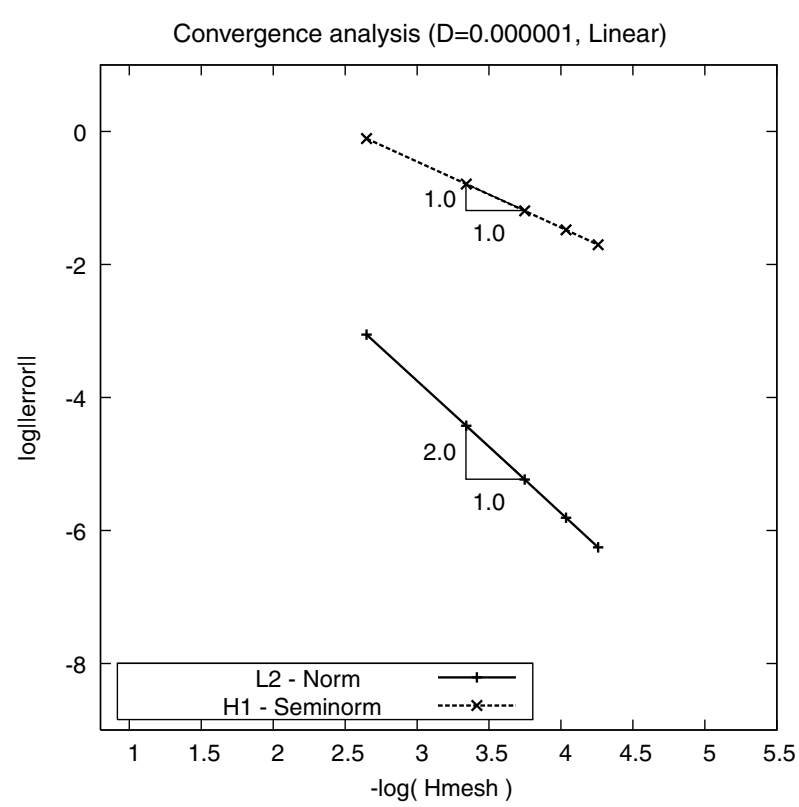

Fig. 20. Linear elements.

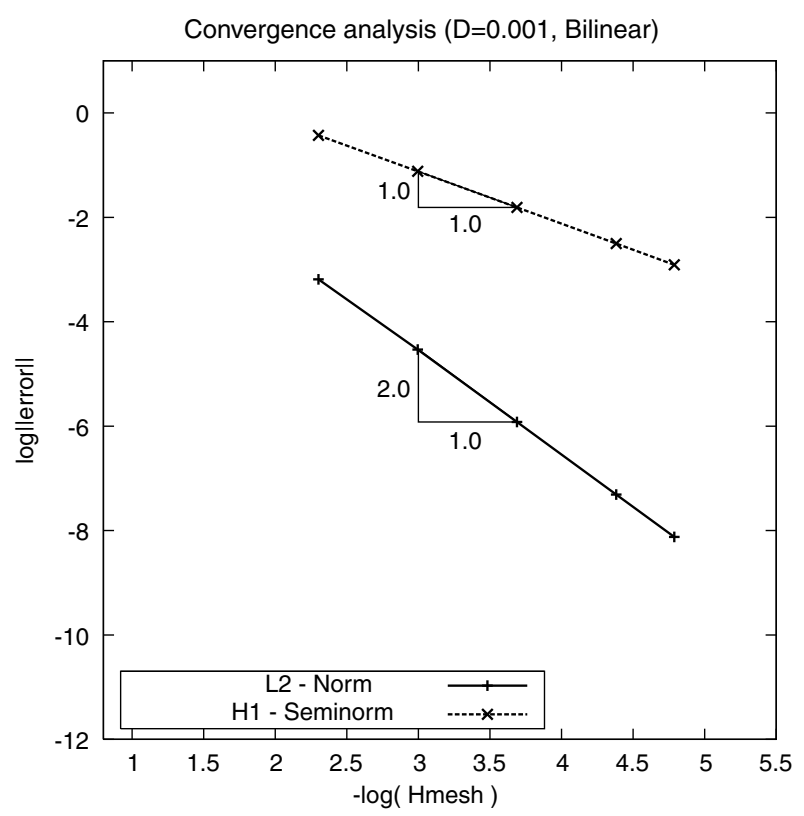

Fig. 21. Bilinear elements. 
$40,80 \times 80$ and $120 \times 120$ and for triangular meshes with 400 , $1600,3600,6400$ and 10,000 elements. The convergence results are presented in graphs $-\log ($ Hmesh $) \times \log \|$ Error $\|$ in the natural basis, where Hmesh $=(\text { ne })^{\frac{1}{2}}$, $\|$ Error $\|=\| \phi-\phi^{h} \|_{L^{2}(\Omega)}$ or $\|$ Error $\|=$ $\left|\phi-\phi^{h}\right|_{H^{1}(\Omega)}$ with ne denoting the number of elements, $n$ the dimension of the domain and $|\circ|_{H^{1}(\Omega)}$ the $\mathrm{H}^{1}$-semi-norm. The quadrilateral mesh $20 \times 20$ is shown in the Fig. 5 and the triangular mesh with 800 elements in the Fig. 6. The convergence study was performed for different values of the diffusion coefficient, e.g., $D=1, D=10^{-3}$ and $D=10^{-6}$. Results for quadrilateral meshes are presented in Figs. 19, 21 and 23 and for triangular meshes are presented in the Figs. 20, 22 and 24, for different values of the diffusion coefficient $D$. Clearly, for all values of $D$ tested, the GPR method presents optimum rates of convergence.

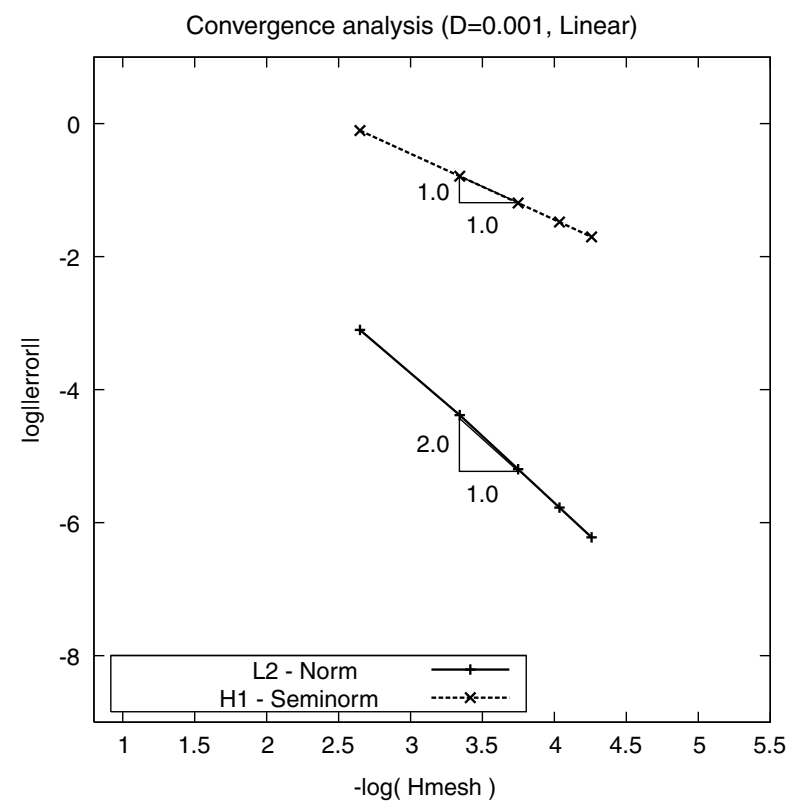

Fig. 22. Linear elements.

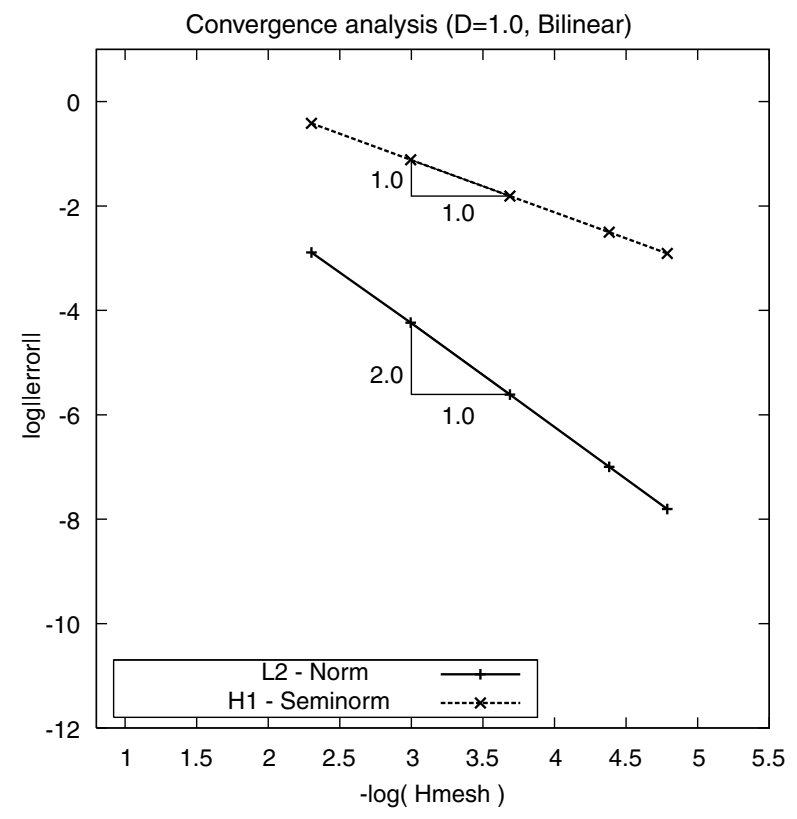

Fig. 23. Bilinear elements.

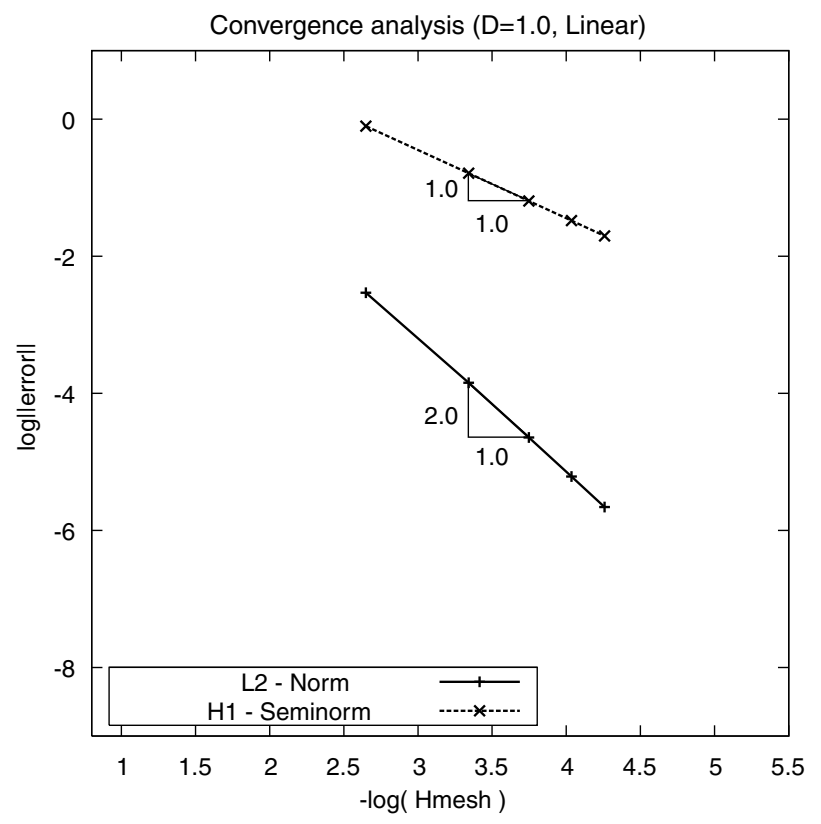

Fig. 24. Linear elements.

\section{Conclusions}

The inability of purely Galerkin formulations on generating robust and stable FEM's capable of handling, for instance, sharp gradients, has pushed the design of the so called stabilized formulations. The present contribution fits within this context. It is devoted to introduce a new consistent FEM to be applied to diffusion-reaction boundary value problems. It is built upon a generic methodology, refereed to as GPR due its main characteristics, which permits to obtain a family of methods that depend on the choice of the GPR-generating matrix and on the $\widehat{\mathscr{L}}$ operator at element level. The GPR-generating matrix encodes information about features of the exact solution to be pursued. The formulation is valid for any space dimension and any order of local basis functions. For each operator and local approximation space the GPR method introduces a maximum number of free parameters via multiple projections of the residual at element level. These free parameters can be determined by appropriate criteria allowing to find the solution in the continuous finite dimensional space $S^{h}$ that best approximates the exact solution. The element matrix of several stabilized methods (as, for example, the methods presented in Refs. $[37,38,43])$ can be obtained departing from GPR method taking into consideration appropriate choices of parameters.

Using the proposed methodology, a new stabilized method of the class GPR was developed for diffusion-reaction problems requiring extra computational effort when compared to typical Galerkin formulations. This extra effort is handled with a pre-processing, not representing a real burden, restricted to the elements $\Omega_{e}$ such that $\Gamma_{e} \cap\left(\Gamma \cup \Gamma_{\mathrm{int}}\right) \neq \varnothing$. The GPR framework is able to retrieve well explored stabilized finite element formulations like the GLS and these can be cast as derivations from the abstract multiscale approach (VMS) introduced in [15]. We believe that there is a connection between GPR and multiscale approaches that should be explored in the near future.

A comprehensive number of numerical experiments was undertaken in order to assess and analyze the proposed method. They clearly indicate that the new method possesses a great performance in terms of accuracy and of stability which compensates the extra computational effort. Moreover, it was possible to embed in generating matrix the mesh distortion what we are convinced 
helped on reducing the sensitivity of the solution to mesh distortions frequently found in real applications.

The presented results are representative as they deal with both regular situations and some presenting sharp layers. A study with a typical regular problem indicates that the proposed method presents optimum convergence rates. Boundary layers were captured with high precision as well.

It should be observed, that the methodology GPR can be used to obtain different stabilized formulations with even better performance when compared to the one introduced here, once a more efficient GPR-generating matrix can be proposed. In [64] the GPR method was applied to Helmholtz equation and as result was obtained a finite element formulation with minimal pollution. The good performance of the proposed formulation obtained for diffusion-reaction problem and Helmholtz equation, stimulates to apply the GPR methodology to other problems in future works, for example, non linear problems, Helmholtz problems in non structured meshes, advection problems and vector systems.

\section{Acknowledgements}

The authors wish to thank the Brazilian research funding agencies CNPq and FAPERJ for their support to this work. Along the final phase of preparation of the present manuscript, F.A. Rochinha was Visiting Professor at the Institute of Applied Mathematics, University of British Columbia, Canada.

\section{References}

[1] R.A. Adams, Sobolev Spaces, Academic Press, New York, 1975.

[2] A.N. Brooks, T.J.R. Hughes, Streamline upwind/Petrov-Galerkin formulations for convection dominated flows with particular emphasis on the incompressible Navier-Stokes equations, Comput. Methods Appl. Mech. Engrg. 32 (1982) 199259.

[3] T.J.R. Hughes, T.E. Tezduyar, Finite element methods for first-order hyperbolic systems with particular emphasis on the compressible Euler equations, Comput. Methods Appl. Mech. Engrg. 45 (1984) 217-284.

[4] C. Johnson, U. Nävert, J. Pitkaranta, Finite element methods for linear hyperbolic problems, Comput. Methods Appl. Mech. Engrg. 45 (1984) 285312.

[5] T.J.R. Hughes, M. Mallet, A new finite element formulation for computational fluid dynamics: IV. A discontinuity capturing operator for multidimensional advective-diffusive systems, Comput. Methods Appl. Mech. Engrg. 58 (1986) 329-336.

[6] T.J.R. Hughes, M. Mallet, A. Mizukami, A new finite element formulation for computational fluid dynamics: II. Beyond SUPG, Comput. Methods Appl. Mech. Engrg. 54 (1986) 341-355.

[7] T.E. Tezduyar, Y.J. Park, Discontinuity Capturing finite element formulations for nonlinear convection-diffusion-reaction equations, Comput. Methods Appl. Mech. Engrg. 59 (1986) 307-325.

[8] T.E. Tezduyar, Y.J. Park, H.A. Deans, Finite element procedures for timedependent convection-diffusion-reaction Systems, Int. J. Numer. Methods Fluids 7 (1987) 1013-1033.

[9] A.C. Galeão, E.G.D. Do Carmo, A consistent approximate upwind PetrovGalerkin method for convection-dominated problems, Comput. Methods Appl. Mech. Engrg. 68 (1988) 83-95.

[10] T.J.R. Hughes, L.P. Franca, G.M. Hulbert, A new finite element formulation for computational fluid dynamics: VII. The Galerkin-least-squares method for advective-diffusive equations, Comput. Methods Appl. Mech. Engrg. 73 (1989) $173-189$.

[11] F. Brezzi, M.O. Bristeau, L.P. Franca, M. Mallet, G. Rogé, A relationship between stabilized finite element methods and the Galerkin with bubble functions, Comput. Methods Appl. Mech. Engrg. 96 (1992) 117-129.

[12] T.E. Tezduyar, Stabilized finite element formulations for incompressible flow computations, Adv. Appl. Mech. 28 (1992) 1-44.

[13] C. Baiocchi, F. Brezzi, L.P. Franca, Virtual bubbles and Galerkin-least-squares type methods Ga.L.S, Comput. Methods Appl. Mech. Engrg. 105 (1993) 125141.

[14] G.J. Le Beau, S.E. Ray, S.K. Aliabadi, T.E. Tezduyar, SUPG finite element computation of compressible flows with the entropy and conservation variables formulations, Comput. Methods Appl. Mech. Engrg. 104 (1993) 397-422.

[15] T.J.R. Hughes, Multiscale phenomena: Green's functions the Dirichlet-toNeumann formulation subgrid scale models bubbles and the origins of stabilized methods, Comput. Methods Appl. Mech. Engrg. 127 (1/4) (1995) 387-401.
[16] A.N. Agarwal, P.M. Pinsky, Stabilized element residual methods (SERM): a posteriori error estimation for advection-diffusion equation, J. Comput. Appl. Math. 74 (1996) 3-17.

[17] E. Oñate, Derivation of stabilized equation for numerical solution of advectivediffusive transport and fluid flow problems, Comput. Methods Appl. Mech. Engrg. 151 (1998) 233-265.

[18] V. John, G. Matthies, F. Schieweck, L. Tobiska, A streamline-diffusion method for nonconforming finite element approximations applied to convectiondiffusion problems, Comput. Methods Appl. Mech. Engrg. 166 (1998) 85-97.

[19] Y.T. Shih, H.C. Elman, Modified streamline diffusion schemes for convectiondiffusion problems, Comput. Methods Appl. Mech. Engrg. 174 (1999) 137-151.

[20] B. Fischer, A. Ramage, D.J. Silvester, A.J. Wathen, On parameter choice and interative convergence for stabilised discretisations of advection-diffusion problems, Comput. Methods Appl. Mech. Engrg. 179 (1999) 179-195.

[21] A. Ramage, A multigrid preconditioner for stabilised discretisations of advection-diffusion problems, J. Comput. Appl. Math. 110 (1999) 187-203.

[22] F. Ilinca, J.F. Hétu, D. Pelletier, On stabilized finite element formulations for incompressible advective-diffusive transport and fluid flow problems, Comput. Methods Appl. Mech. Engrg. 188 (2000) 235-255.

[23] V. John, A numerical study of a posteriori error estimators for convectiondiffusion equations, Comput. Methods Appl. Mech. Engrg. 190 (2000) 757781.

[24] A. Papastavrou, Rüdiger Verfürth, A posteriori error estimator for stationary convection-diffusion problems: a computational comparison, Comput. Methods Appl. Mech. Engrg. 189 (2000) 449-462.

[25] T.E. Tezduyar, Y. Osawa, Finite element stabilization parameters computed from element matrices and vectors, Comput. Methods Appl. Mech. Engrg. 190 (2000) 411-430.

[26] C. Farhat, I. Harari, L. Franca, The discontinuous enrichment method, Comput. Methods Appl. Mech. Engrg. 190 (2001) 6455-6479.

[27] A. Nesliturk, I. Harari, The nearly-optimal Petrov-Galerkin method for convection-diffusion problems, Comput. Methods Appl. Mech. Engrg. 192 (2003) 2501-2519.

[28] E.G. Dutra do Carmo, G.B. Alvarez, A new stabilized finite element formulation for scalar convection-diffusion problems: the streamline and approximate upwind/Petrov-Galerkin method, Comput. Methods Appl. Mech. Engrg. 192 (2003) 3379-3396.

[29] T.E. Tezduyar, Computation of moving boundaries and interfaces and stabilization parameters, Int. J. Numer. Methods Fluids 43 (2003) 555-575.

[30] E.G. Dutra do Carmo, G.B. Alvarez, A new upwind function in stabilized finite element formulations using linear and quadratic elements for scalar convection-diffusion problems, Comput. Methods Appl. Mech. Engrg. 193 (2004) 2383-2402.

[31] L.P. Franca, E.G.D. Do Carmo, The Galerkin gradient least squares method, Comput. Methods Appl. Mech. Engrg. 74 (1989) 41-54.

[32] L.P. Franca, C. Farhat, Bubble functions prompt unusual stabilized finite element methods, Comput. Methods Appl. Mech. Engrg. 123 (1995) 299-308.

[33] S. Idelsohn, N. Nigro, M. Storti, G. Buscaglia, A Petrov-Galerkin formulation for advection-reaction-diffusion problems, Comput. Methods Appl. Mech. Engrg. 136 (1996) 27-46.

[34] J. Li, I.M. Navon, Uniformly convergent finite element methods for singularly perturbed elliptic boundary value problems I: reaction-diffusion type, Comput. Math. Appl. 35 (1998) 57-70.

[35] Ramon Codina, Comparison of some finite element methods for solving the diffusion-convection-reaction equation, Comput. Methods Appl. Mech. Engrg. 156 (1998) 185-210.

[36] Robert Sandboge, Adaptive finite element methods for systems of reactiondiffusion equations, Comput. Methods Appl. Mech. Engrg. 166 (1998) 309328.

[37] Ramon Codina, On stabilized finite element methods for linear systems of convection-diffusion-reaction equations, Comput. Methods Appl. Mech. Engrg. 188 (2000) 61-82.

[38] L.P. Franca, F. Valentin, On an improved unusual stabilized finite element method for the advective-reactive-diffusive equation, Comput. Methods Appl. Mech. Engrg. 190 (2000) 1785-1800.

[39] Ramon Codina, A stabilized finite element method for generalized stationary incompressible flows, Comput. Methods Appl. Mech. Engrg. 190 (2001) 26812706.

[40] Jouni Freund, The space-continuous-discontinuous Galerkin method, Comput. Methods Appl. Mech. Engrg. 190 (2001) 3461-3473.

[41] G. Hauke, A.G. Olivares, Variational subgrid scale formulations for the advection-diffusion-reaction equation, Comput. Methods Appl. Mech. Engrg. 190 (2001) 6847-6865.

[42] Jichun Li, Uniform convergence of discontinuous finite element methods for singularly perturbed reaction-diffusion problems, Comput. Math. Appl. 44 (2002) 231-240.

[43] G. Hauke, A simple subgrid scale stabilized method for the advectiondiffusion-reaction equation, Comput. Methods Appl. Mech. Engrg. 191 (2002) 2925-2947.

[44] Peter K. Moore, Implicit interpolation error-based error estimation for reaction-diffusion equations in two space dimensions, Comput. Methods Appl. Mech. Engrg. 192 (2003) 4379-4401.

[45] Erik Burman, Peter Hansbo, Edge stabilization for Galerkin approximations of convection-diffusion-reaction problems, Comput. Methods Appl. Mech. Engrg. 193 (2004) 1437-1453. 
[46] Leopoldo P. Franca, Alexandre L. Madureira, Frederic Valentin, Towards multiscale functions: enriching finite element spaces with local but not bubble-like functions, Comput. Methods Appl. Mech. Engrg. 194 (2005) 30063021.

[47] Albert Romkes, Serge Prudhomme, J. Tinsley Oden, Convergence analysis of a discontinuous finite element formulation based on second order derivatives, Comput. Methods Appl. Mech. Engrg. 195 (2006) 3461-3482.

[48] M. Parvazinia, V. Nassehi, Multiscale finite element modeling of diffusionreaction equation using bubble functions with bilinear and triangular elements, Comput. Methods Appl. Mech. Engrg. 196 (2007) 1095-1107.

[49] D. Lacasse, A. Garon, D. Pelletier, Development of an adaptive discontinuousGalerkin finite element method for advection-reaction equations, Comput. Methods Appl. Mech. Engrg. 196 (2007) 2071-2083.

[50] I. Harari, T.J.R. Hughes, Finite element method for the Helmholtz equation in an exterior domain: model problems, Comput. Methods Appl. Mech. Engrg. 87 (1991) 59-96.

[51] I. Harari, T.J.R. Hughes, Galerkin/least squares finite element methods for the reduced wave equation with non-reflecting boundary conditions in unbounded domains, Comput. Methods Appl. Mech. Engrg. 98 (1992) 411454.

[52] F. Ihlenburg, I. Babuška, Finite element solution of the Helmholtz equation with high wave number Part I: the h-version of the FEM, Comput. Math. Appl. 30 (9) (1995) 9-37.

[53] L.L. Thompson, P.M. Pinsky, A Galerkin least squares finite element method for the two-dimensional Helmholtz equation, Int. J. Numer. Methods Engrg. 38 (3) (1995) 371-397.

[54] I. Babuška, F. Ihlenburg, E.T. Paik, S.A. Sauter, A generalized finite element method for solving the Helmholtz equation in two dimensions with minimal pollution, Comput. Methods Appl. Mech. Engrg. 128 (1995) 325-359.

[55] L.P. Franca, C. Farhat, A.P. Macedo, M. Lesoinne, Residual-free bubbles for the Helmholtz equation, Int. J. Numer. Methods Engrg. 40 (1997) 4003-4009.
[56] F. Ihlenburg, Finite Element Analysis of Acoustic Scattering, Applied Mathematical Sciences, vol. 132, Springer-Verlag, New York, 1998.

[57] L.P. Franca, A.P. Macedo, A two-level finite element method and its application to the Helmholtz equation, Int. J. Numer. Methods Engrg. 43 (1) (1998) 23-32

[58] A.A. Oberai, P.M. Pinsky, A residual-based finite element method for the Helmholtz equation, Int. J. Numer. Methods Engrg. 49 (2000) 399-419.

[59] C. Farhat, A.P. Macedo, M. Lesoinne, A two-level domain decomposition method for the iterative solution of high frequency exterior Helmholtz problems, Numer. Math. 85 (2000) 283-308.

[60] C. Farhat, I. Harari, U. Hetmaniuk, A discontinuous Galerkin method with Lagrange multipliers for the solution of Helmholtz problems in the midfrequency regime, Comput. Methods Appl. Mech. Engrg. 192 (2003) 13891419.

[61] L.L. Thompson, P.M. Pinsky, in: E. Stein, R. de Borst, T.J.R. Hughes (Eds.) Acoustics: Encyclopedia of Computational Mechanics, Jonh Wiley \& Sons, Ltd., 2004.

[62] G.B. Alvarez, A.F.D. Loula, E.G. Dutra do Carmo, F.A. Rochinha, A discontinuous finite element formulation for the Helmholtz equation, Comput. Methods Appl. Mech. Engrg. 195 (2006) 4018-4035.

[63] A.F.D. Loula, G.B. Alvarez, E.G. Dutra do Carmo, F.A. Rochinha, A discontinuous finite element method at element level for Helmholtz equation, Comput. Methods Appl. Mech. Engrg. 196 (2007) 867-878.

[64] E.G. Dutra do Carmo, G.B. Alvarez, A.F.D. Loula, F.A. Rochinha, A nearly optimal Galerkin Projected Residual finite element method for Helmholtz problem, Comput. Methods Appl. Mech. Engrg. 197 (13-16) (2008) 1362-1375.

[65] F. Valentin, L.P. Franca, Combining stabilized finite element methods, Comput. Appl. Math. 14 (3) (1995) 285-300.

[66] G. Hauke, G. Sangalli, M.H. Doweidar, Combining adjoint stabilized methods for the advection-diffusion-reaction problem, Math. Models Methods Appl. Sci. 17 (2) (2007) 305-326. 